\title{
Properties of Synaptically Evoked Astrocyte Calcium Signal Reveal Synaptic Information Processing by Astrocytes
}

\author{
Gertrudis Perea and Alfonso Araque \\ Instituto Cajal, Consejo Superior de Investigaciones Científicas, Madrid 28002, Spain
}

The synaptic control of the astrocytic intracellular $\mathrm{Ca}^{2+}$ is crucial in the reciprocal astrocyte-neuron communication. Using electrophysiological and $\mathrm{Ca}^{2+}$ imaging techniques in rat hippocampal slices, we investigated the astrocytic $\mathrm{Ca}^{2+}$ signal modulation induced by synaptic terminals that use glutamate and acetylcholine. $\mathrm{Ca}^{2+}$ elevations were evoked by glutamate released from Schaffer collaterals and by acetylcholine, but not glutamate, released by alveus stimulation, indicating that astrocytes discriminate the activity of different synapses belonging to different axon pathways. The $\mathrm{Ca}^{2+}$ signal was modulated bidirectionally by simultaneous activation of both pathways, being depressed at high stimulation frequencies and enhanced at low frequencies. The $\mathrm{Ca}^{2+}$ modulation was attributable to astrocytic intrinsic properties, occurred at discrete regions of the processes, and controlled the intracellular expansion of the $\mathrm{Ca}^{2+}$ signal. In turn, astrocyte $\mathrm{Ca}^{2+}$ signal elicited NMDA receptor-mediated currents in pyramidal neurons. Therefore, because astrocytes discriminate and integrate synaptic information, we propose that they can be considered as cellular elements involved in the information processing by the nervous system.

Key words: astrocytes; intracellular calcium; neurotransmitter release; glutamate; synaptic activity; neuron-glia interaction

\section{Introduction}

Evidence obtained during the last few years has established a new concept of the synaptic physiology, the tripartite synapse, in which astrocytes play an active role by exchanging information with the synaptic elements (Araque et al., 1999; Carmignoto, 2000; Haydon and Araque, 2002; Volterra and Bezzi, 2002; Auld and Robitaille, 2003; Newman, 2003). This concept is based on the demonstration that astrocytes display a form of excitability based on intracellular $\mathrm{Ca}^{2+}$ variations (Pasti et al., 1997; Verkhratsky et al., 1998; Haydon, 2001; Charles and Giaume, 2002; Haydon and Araque, 2002; Nedergaard et al., 2003), respond to synaptically released neurotransmitters (Porter and McCarthy, 1996; Pasti et al., 1997; Grosche et al., 1999; Latour et al., 2001; Araque et al., 2002), and modulate neuronal excitability and synaptic transmission by releasing neuroactive substances through, at least some of them, $\mathrm{Ca}^{2+}$-dependent mechanisms (Araque et al., 1998a,b; Kang et al., 1998; Newman and Zahs, 1998; Robitaille, 1998; Parri et al., 2001; Beattie et al., 2002; Brockhaus and Deitmer, 2002; Volterra and Bezzi, 2002; Newman, 2003; Zhang et al., 2003; Fiacco and McCarthy, 2004; Liu et al., 2004).

In this loop of information exchange between astrocytes and neurons, the synaptic control of the astrocytic $\mathrm{Ca}^{2+}$ is a key element. However, the properties and regulation of the synaptically

Received Sept. 24, 2004; revised Jan. 11, 2005; accepted Jan. 13, 2005.

This work was supported by grants from the Ministerio de Ciencia y Tecnología (BFI2001-0206) and Ministerio de Educación y Ciencia (BFU2004-00448), Spain. G.P. is a Consejo Superior de Investigaciones Científicas predoctoral fellow. We thank Drs. W. Buño, P. G. Haydon, J. Lerma, and E. D. Martín for helpful suggestions and comments on drafts of this manuscript.

Correspondence should be addressed to Dr. Alfonso Araque, Instituto Cajal, Doctor Arce 37, Madrid 28002, Spain. E-mail: araque@cajal.csic.es.

DOI:10.1523/JNEUROSCI.3965-04.2005

Copyright $\odot 2005$ Society for Neuroscience $\quad$ 0270-6474/05/252192-13\$15.00/0 evoked astrocytic $\mathrm{Ca}^{2+}$ signal are essentially unknown. We investigated the modulation of the $\mathrm{Ca}^{2+}$ signal of hippocampal astrocytes induced by activation of two different afferent pathways that use glutamate and acetylcholine ( $\mathrm{ACh}$ ) as neurotransmitters. We found that although stimulation of glutamatergic Schaffer collaterals (SC) elicited $\mathrm{Ca}^{2+}$ elevations selectively mediated by glutamate receptors (GluRs), stimulation of the alveus, which contains both cholinergic and glutamatergic axons, evoked glutamate transporter currents as well as ACh- but not glutamate-mediated $\mathrm{Ca}^{2+}$ elevations, indicating that astrocytes discriminate between the activity of different synaptic terminals belonging to different axon pathways.

Furthermore, we found that the astrocytic $\mathrm{Ca}^{2+}$ was modulated by interaction of those synaptic inputs (i.e., SC and alveus) because of intrinsic properties of astrocytes and that this regulation was bidirectional, being potentiated or depressed depending on the level of synaptic activity. $\mathrm{Ca}^{2+}$ responses examined at subcellular regions in astrocytic processes showed similar modulation. Furthermore, the synaptic interaction controlled the propagation of the astrocyte intracellular $\mathrm{Ca}^{2+}$ signal.

Although the ability of astrocytes to release glutamate through a $\mathrm{Ca}^{2+}$-dependent mechanism is well established (Bezzi et al., 1998, 2004; Araque et al., 2000; Parpura and Haydon, 2000; Pasti et al., 2001; Zhang et al., 2004), its consequences on neuronal physiology are not completely determined. We investigated the electrophysiological effects of the astrocyte $\mathrm{Ca}^{2+}$ signal on CA1 pyramidal neurons, and we found that astrocyte $\mathrm{Ca}^{2+}$ elevations evoked NMDA receptor (NMDAR)-mediated slow inward currents (SICs) in neurons.

Therefore, the present results show that the astrocytic $\mathrm{Ca}^{2+}$ signal does not simply reflect synaptic activity but that astrocytes are able to discriminate between the activity of different synapses and to integrate those inputs attributable to the existence of cel- 
lular intrinsic properties, indicating that astrocytes show integrative properties for synaptic information processing.

\section{Materials and Methods}

Hippocampal slice preparation. Acute hippocampal slices were obtained as described previously (Araque et al., 2002). All of the procedures for handling and killing animals followed European Commission guidelines (86/609/CEE) and were supervised by the Instituto Cajal veterinary officer. Briefly, Wistar rats (12-17 d old) were anesthetized and then decapitated. The brain was removed rapidly and placed in ice-cold artificial CSF (ACSF). Brain slices (350-450 $\mu \mathrm{m}$ thick) were cut with a Vibratome and incubated for $>1 \mathrm{~h}$ at room temperature $\left(21-24^{\circ} \mathrm{C}\right)$ in ACSF. The ACSF contained (in mM) $124 \mathrm{NaCl}, 2.69 \mathrm{KCl}, 1.25 \mathrm{KH}_{2} \mathrm{PO}_{4}, 2 \mathrm{MgSO}_{4}, 26$ $\mathrm{NaHCO}_{3}, 2 \mathrm{CaCl}_{2}$, and 10 glucose and was gassed with $95 \% \mathrm{O}_{2} / 5 \% \mathrm{CO}_{2}$, $\mathrm{pH}$ 7.3. Slices were then transferred to an immersion recording chamber and superfused with gassed ACSF. In some cases, the ACSF contained $100 \mu \mathrm{M} 4$-aminopyridine (4-AP) to enhance astrocytic responses (Porter and McCarthy, 1996; Araque et al., 2002). In experiments designed to optimize NMDAR activation, the extracellular $\mathrm{Mg}^{2+}$ was equimolarly substituted by $\mathrm{Ca}^{2+}$, and $10 \mu \mathrm{m}$ glycine was added. Cells were visualized under an Olympus BX50WI microscope (Olympus Optical, Tokyo, Japan) equipped with infrared and differential interference contrast imaging devices and with a $40 \times$ water immersion objective.

Electrophysiology. Simultaneous fluorescence photometric $\mathrm{Ca}^{2+}$ measurements and electrophysiological recordings from astrocytes located in the stratum oriens of the CA1 hippocampal region were made using the whole-cell patch-clamp technique. Patch electrodes were fabricated from borosilicate glass capillaries and had resistances of 6-10 $\mathrm{M} \Omega$ when filled with the internal solution that contained the following (in mM): 100 $\mathrm{KMeSO}_{4}, 50 \mathrm{KCl}, 10 \mathrm{HEPES}$, and $4 \mathrm{ATP}-\mathrm{Na}_{2}$, $\mathrm{pH}$ 7.3. Recordings were obtained with an Axoclamp-2A amplifier (Axon Instruments, Foster City, $\mathrm{CA}$ ) in the continuous single-electrode voltage-clamp mode or a PC-ONE amplifier (Dagan Instruments, Minneapolis, MN). Fast and slow whole-cell capacitances were neutralized, series resistance was compensated $(\sim 70 \%)$, and the membrane potential was held at resting potential. Signals were fed to a Pentium-based personal computer through a DigiData 1320 interface board (Axon Instruments). pClamp 8 software (Axon Instruments) was used for stimulus generations, data display, acquisition, and storage.

Astrocytes were identified according to morphological and electrophysiological criteria (Bergles and Jahr, 1997; Pasti et al., 1997; Bezzi et al., 1998; Bergles et al., 2000; Araque et al., 2002). In some cases, the recorded cells were later immunocytochemically studied and examined using laser-scanning confocal microscopy. The dual labeling with fluo-3 and GFAP antibody of the recorded cells confirmed their identification as astrocytes (data not shown) (Araque et al., 2002). Bipolar nichrome wire electrodes ( $80 \mu \mathrm{m}$ diameter) were connected to a stimulator through isolation units (Grass S88; Grass Instruments, Quincy, MA) and placed in the stratum radiatum near the border of the CA1 pyramidal neurons to stimulate SC-commissural afferents; they were also placed in the stratum oriens/alveus near the subiculum area (for simplicity herein termed alveus), which contains cholinergic axons from diagonal band of Broca and septum (Lewis and Shute, 1967). Trains of stimuli at $30 \mathrm{~Hz}$ during $5 \mathrm{~s}$ were delivered at $0.013 \mathrm{~s}^{-1}$, unless stated otherwise. This low-rate train stimulation evoked reliable successive responses (data not shown) (Araque et al., 2002), and in some cases, up to three responses were averaged. In some experiments, either the frequency or the duration of the stimulus was varied, but the stimulation intensities were unchanged throughout the experiment to avoid variations in the amount of fibers stimulated. When the SC and alveus were stimulated independently or simultaneously, the sequence of stimulation was orderless. Because similar results were observed regardless of that sequence, data were pooled together. Experiments were performed at room temperature (21$\left.24^{\circ} \mathrm{C}\right)$. Data are expressed as mean \pm SEM. Statistical differences were established by the Student's $t$ test.

Paired whole-cell recordings from neurons and astrocytes. In some experiments, we performed paired whole-cell recordings from CA1 pyramidal neurons and stratum oriens astrocytes (distance of the somas, $<150 \mu \mathrm{m}$ ) (see Fig. 6A). In some cases, recorded astrocytes were stimulated with trains of $60-180 \mathrm{mV}$ depolarizing pulses (duration, $100-500$ $\mathrm{ms}$ ) delivered every $1 \mathrm{~s}$ for 3-10 min. Mean frequencies of astrocyte $\mathrm{Ca}^{2+}$ elevations and neuronal SICs were evaluated over 3-10 min periods.

Measurement of intracellular $\mathrm{Ca}^{2+}$ variations. $\mathrm{Ca}^{2+}$ levels in single astrocytes were monitored by fluorescence microscopy using the $\mathrm{Ca}^{2+}$ indicator fluo-3. Patch pipettes were filled with the internal solution containing $50 \mu \mathrm{m}$ fluo-3 (Molecular Probes, Eugene, OR). Cells were illuminated with a xenon lamp at $490 \mathrm{~nm}$ using a monochromator Polychrome II (T.I.L.L. Photonics, Planegg, Germany). Fluorescence intensity was collected by a photomultiplier tube (model R928; Hamamatsu Photonic System, Bridgewater, NJ) from a variable rectangular window (side, 25-50 $\mu \mathrm{m}$ ) containing the soma of the recorded astrocyte. Cells were illuminated during $20-200 \mathrm{~ms}$ every $0.5-1 \mathrm{~s}$, and the collected fluorescence signal was integrated using the T.I.L.L. Photonics photometry system. $\mathrm{Ca}^{2+}$ variations were estimated as changes in the fluorescence signal over baseline $\left(\Delta F / F_{0}\right)$ after background subtraction. Astrocytes were considered to respond to the stimulation when the fluorescence signal increased two times above the SD of the basal signal.

$\mathrm{Ca}^{2+}$ imaging. In some cases, slices were incubated with fluo-3 AM (2-5 $\mu$ l of $2 \mathrm{~mm}$ dye was dropped over the hippocampus, attaining a final concentration of 2-10 $\mu \mathrm{M}$ ) for 20-30 min at room temperature. In these conditions, most of the cells loaded were astrocytes (Kang et al., 1998; Parri et al., 2001; Aguado et al., 2002; Nett et al., 2002; Liu et al., 2004), as confirmed in some cases by their electrophysiological properties (see Fig. $7 A$, in which fluorescence of the pyramidal layer was very low because of poor neuronal loading). Astrocytes were imaged using a CCD camera (Retiga EX; Qimaging, Burnaby, British Columbia, Canada) attached to the Olympus microscope. Cells were illuminated during 200-500 ms, and images were acquired every 1-2 s. The monochromator Polychrome II and the CCD camera were controlled and synchronized by customized software (Solex Consultores SL, Madrid, Spain). Quantitative epifluorescence measurements were made using ImageJ public domain software (developed at the National Institutes of Health). $\mathrm{Ca}^{2+}$ variations were estimated as $\Delta F / F_{0}$ after background subtraction, and regions of interest were considered to respond to the stimulation when $\Delta F / F_{0}$ increased $>5 \%$ for at least two consecutive images with a delay $\leq 15 \mathrm{~s}$ after the stimulation. Although nerve stimulation evoked long-lasting, transient $\mathrm{Ca}^{2+}$ elevations (i.e., $\mathrm{Ca}^{2+}$ spikes) (see Fig. $1 C$ ), direct depolarization of astrocytes evoked repetitive $\mathrm{Ca}^{2+}$ spikes (i.e., $\mathrm{Ca}^{2+}$ oscillations) (see Fig. $6 E$ ). The parameter used to quantify the astrocyte $\mathrm{Ca}^{2+}$ signal was the amplitude of the transient $\mathrm{Ca}^{2+}$ spikes. In addition, in the experiments reported in Figure 6, we analyzed the frequency of the astrocyte $\mathrm{Ca}^{2+}$ elevations that occurred spontaneously or after membrane depolarization. Intracellular $\mathrm{Ca}^{2+}$ elevations were observed in astrocytes either spontaneously (Latour et al., 2001; Aguado et al., 2002) (see Fig. 6) or after SC stimulation (see Results). Those astrocytes in which these $\mathrm{Ca}^{2+}$ oscillations prevented the unambiguous analysis of the nerve-evoked responses were not considered further.

A concern about the methodology used (short loading times of fluo-3 $\mathrm{AM}$ ) relates to the high surface loading of the slices, which could result in a preferential loading of superficial damaged astrocytes and high background fluorescence that could mask $\mathrm{Ca}^{2+}$ fluorescence changes. Incubating the slices for $3 \mathrm{~h}$ and in the presence of pluronic acid $(0.001 \%)$ did not improve the depth of loading, as examined by laser-scanning confocal microscopy ( $43 \pm 4$ and $45 \pm 3 \mu \mathrm{m}$ in five and three slices incubated for $30 \mathrm{~min}$ and $3 \mathrm{~h}$, respectively). Hence, some slices were incubated with fluorescent dyes that offer better resolution [i.e., fluo-4 AM $(2-10 \mu \mathrm{M})$, Calcium Green-1 AM $(10 \mu \mathrm{M})$, and fura-2 AM $(10 \mu \mathrm{M})]$ and provided similar results to those obtained with fluo-3 ( $n \geq 25$ astrocytes from at least three slices in each condition; data not shown). Whole-cell recordings of astrocytes from either unloaded or fluo-3 AM-loaded slices showed similar membrane resting potential $(-72.7 \pm 0.9$ and $-70.2 \pm 1.6 \mathrm{mV} ; n=10)$ and conductance $(32.4 \pm 3.6$ and $37.9 \pm 7.4 \mathrm{nS})$, suggesting that $\mathrm{Ca}^{2+}$ signal recordings were likely obtained from undamaged astrocytes. Moreover, similar results were obtained from either fluo-3 AM- or whole-cell-loaded astrocytes, which were not necessarily superficial. Together, these results indicate that astrocyte $\mathrm{Ca}^{2+}$ signal discrimination and modulation were unlikely because of technical limitations.

Ionophoresis. ACh and glutamate were ionophoretically delivered by 1-5 s duration current pulses (MicroIontophoresis Dual Current Pulse 
Generator; World Precision Instruments, Sarasota, FL) using a pulled theta capillary, and each compartment was filled separately with ACh $(0.5$ $\mathrm{M}$ in ACSF, pH 6) and Glu (0.7 $\mathrm{m}$ in ACSF, $\mathrm{pH} 8$ ). Likewise, ATP (20 mm in ACSF, pH 7.5-8) was applied. Ionophoretical experiments were performed in $1 \mu \mathrm{M}$ tetrodotoxin (TTX).

Drugs. (S)- $\alpha$-methyl-4-carboxyphenylglycine (MCPG), 6-cyano-7-nitroquinoxaline-2,3dione (CNQX), $\mathrm{D}(-)$-2-amino-5-phosphonopentanoic acid (AP-5), and L-trans-pyrrolidine2,4-dicarboxylate (t-PDC) were purchased from Tocris Cookson (Bristol, UK); dihydrokainate (DHK) was purchased from Ocean Produce International (Shelburne, Nova Scotia, Canada). All other drugs were from Sigma (St. Louis, MO).

\section{Results}

Astrocytic responses evoked by the activity of SC synaptic terminals

We recently showed that hippocampal stratum oriens astrocytes responded with $\mathrm{Ca}^{2+}$ elevations to ACh but not to glutamate released after stimulation of the alveus, which contains cholinergic and glutamatergic axons (Araque et al., 2002). Because those astrocytes express functional GluRs (Porter and McCarthy, 1996; Shelton and McCarthy, 1999; Latour et al., 2001; Araque et al., 2002), we investigated whether they responded to a different glutamatergic input (i.e., the SC, which projects to the stratum radiatum and stratum oriens of the CA1 hippocampal region) (Li et al., 1994). We simultaneously recorded intracellular $\mathrm{Ca}^{2+}$ levels and whole-cell currents of morphologically and electrophysiologically identified astrocytes in the stratum oriens of the CA1 hippocampal area (Fig. 1 $A, B$ ). Electrical stimulation of SCs evoked long-lasting inward currents $(-39.9 \pm 5.5 \mathrm{pA} ; n=31)$ and transient intracellular $\mathrm{Ca}^{2+}$ elevations (in $84 \%$ of the astrocytes, from a sample of 31 cells) (Fig. 1C). The amplitude of both responses depended on the frequency and duration of the stimulus (data not shown).

In addition to the nerve-evoked transient $\mathrm{Ca}^{2+}$ elevations, astrocytes also displayed $\mathrm{Ca}^{2+}$ oscillations that occurred spontaneously (Latour et al., 2001; Aguado et al., 2002) (see Fig. 6) and that could be elicited by nerve stimulation. These $\mathrm{Ca}^{2+}$ oscillations were modulated by the SC stimulation, increasing their frequency from $0.2 \pm 0.1$ to $0.8 \pm 0.1 \mathrm{~min}^{-1}$ before and after the stimulation, respectively ( $n=41$ astrocytes; data not shown). The present work focused on the transient $\mathrm{Ca}^{2+}$ elevations, and the stimulation-evoked $\mathrm{Ca}^{2+}$ oscillations were not analyzed further.

To investigate whether these responses were attributable to synaptically released neurotransmitters, we used pharmacological tools that modulate nerve activity and neurotransmitter release (Fig. $1 D-G$ ). The SC-induced $\mathrm{Ca}^{2+}$ elevations and inward currents were abolished by the sodium channel antagonist TTX $(1 \mu \mathrm{M} ; n=8)$, which prevents action potential generation, and by extracellular $\mathrm{Cd}^{2+}(100 \mu \mathrm{M} ; n=6)$, which blocks the $\mathrm{Ca}^{2+}$ influx test at ${ }^{*} p<0.05$ and ${ }^{\#} p<0.001$.
B

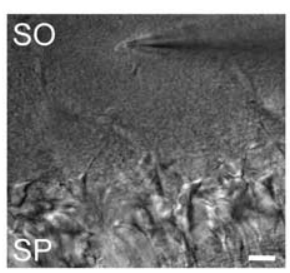

C

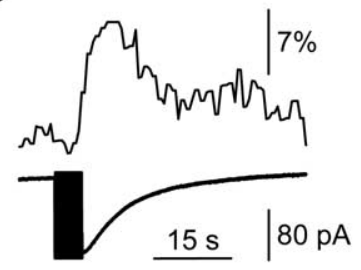

Figure 1. Responses evoked by SC stimulation in hippocampal stratum oriens (SO) astrocytes. $\boldsymbol{A}$, Schematic drawing of the recorded astrocyte in the stratum oriens. Note the recording pipette on the right side of the astrocyte. Scale bar, $20 \mu \mathrm{m}$. $\boldsymbol{C}$ Representative astrocytic $\mathrm{Ca}^{2+}$ levels (top trace) and whole-cell membrane currents (bottom trace) elicited by SC stimulation ( 30 recordings of the membrane current amplitude and fluorescence intensity, respectively, evoked by $S C$ stimulation in the presence f $100 \mu \mathrm{M} 4-\mathrm{AP}(n=21), 100 \mu \mathrm{M} \mathrm{Cd}^{2+}(n=6), 1 \mu \mathrm{M}$ TTX $(n=8), 0.3 \mathrm{~mm}$ t-PDC plus $1 \mathrm{~mm}$ DHK $(n=8), 20 \mu \mathrm{m}$ CNQX plus 50 $\mu \mathrm{M}$ AP-5 plus $0.8 \mathrm{~mm} \mathrm{MCPG}(n=8)$, and $1 \mu \mathrm{m}$ thapsigargin $(n=5)$. Significant differences were established by the Student's

through voltage-gated $\mathrm{Ca}^{2+}$ channels required for evoked neurotransmitter release. Furthermore, 4-AP (100 $\mu \mathrm{M} ; n=21)$, a potassium channel blocker that enhances the transmitter release, increased both the astrocytic $\mathrm{Ca}^{2+}$ elevations and the inward currents elicited by SC stimulation. These results indicate that the astrocytic responses were evoked by neurotransmitter release from synaptic terminals.

Astrocytes express glutamate transporters responsible for the clearance of glutamate from the extracellular space (Mennerick et al., 1996; Bergles et al., 1999). It is known that glutamate released from SCs can activate nearby stratum radiatum hippocampal astrocytes generating a net inward membrane current attributable to the electrogenic uptake of glutamate (Bergles and Jahr, 1997). We tested whether the SC-induced inward current in stratum oriens astrocytes was attributable to activation of glutamate transporters. The amplitude of the inward current was reduced after superfusion with both $1 \mathrm{~mm}$ DHK (a selective inhibitor of 
A
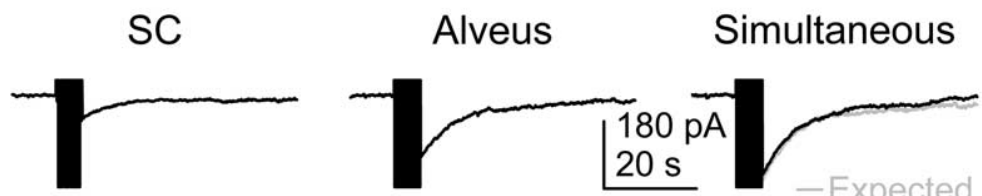

B
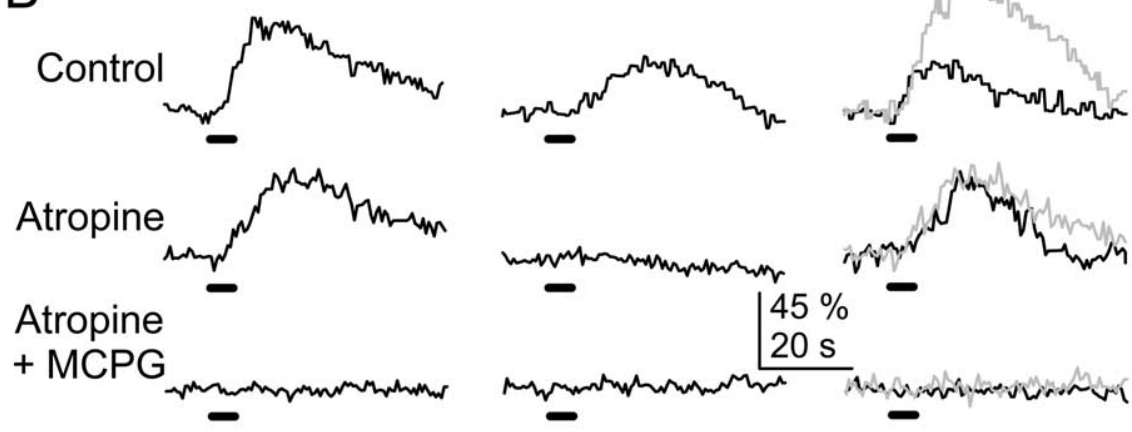

C
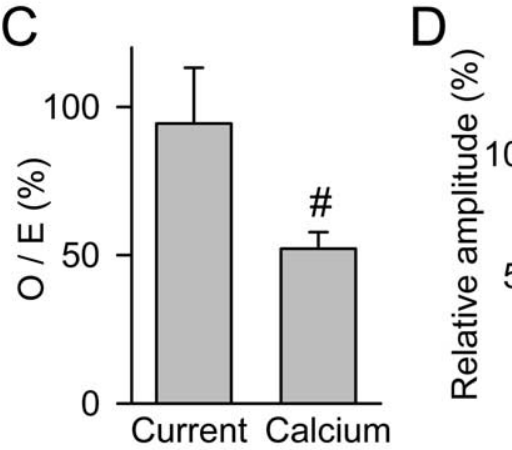

$\mathrm{E}$

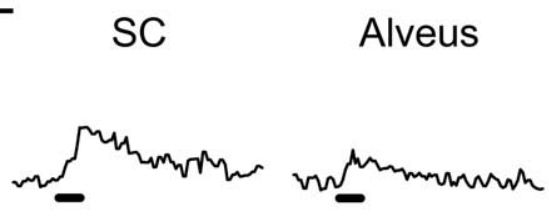

Alveus

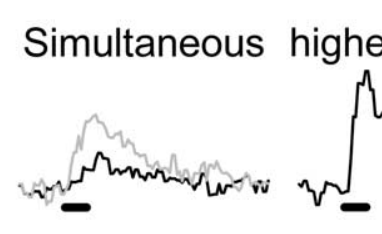

Figure 2. The astrocyte $\mathrm{Ca}^{2+}$ signal was modulated by the simultaneous activity of alveus and SC synaptic terminals. $\boldsymbol{A}$, Representative whole-cell currents elicited by independent or simultaneous stimulation of the $\mathrm{SC}$ and alveus ( $30 \mathrm{~Hz}, 5 \mathrm{~s})$. In a simultaneous stimulation condition, black and gray traces correspond to the observed and expected responses (i.e., the summation of responses evoked by independent stimulation of both pathways), respectively, as in all other figures. $\boldsymbol{B}$, Astrocyte $\mathrm{Ca}^{2+}$ levels evoked by independent or simultaneous stimulation of the $S C$ and alveus in control conditions, in the presence of $50 \mu \mathrm{m}$ atropine, and after the addition of $0.8 \mathrm{mM} \mathrm{MCPG}$. Horizontal lines at the bottom of each trace represent the stimuli, as in all other figures. $\boldsymbol{C}$, Ratio between observed and expected responses. $\boldsymbol{D}$, Relative amplitude of the astrocytic $\mathrm{Ca}^{2+}$ elevations evoked by independent or simultaneous stimulation of the $S C$ and alveus in controls and in the presence of $50 \mu \mathrm{m}$ atropine and $0.8 \mathrm{~mm} \mathrm{MCPG}$. The responses evoked by simultaneous stimulation were quantified from the 0/E ratio (i.e., the ratio between the observed response evoked by simultaneous stimulation and the expected response: the summation of the responses evoked by independent stimulation) (see Results). $n \geq 5$ for each bar. $\boldsymbol{E}$, Representative example of $\mathrm{Ca}^{2+}$ elevations evoked by the SC, alveus, simultaneous stimulation at a constant intensity, and after increasing the SC stimulation intensity. Note that the $\mathrm{Ca}^{2+}$ elevation elicited by SC stimulation at a stronger intensity was higher than the expected $\mathrm{Ca}^{2+}$ increase under simultaneous stimulation (gray trace). Significant differences were established by the Student's $t$ test at ${ }^{\#} p<0.001$.

GLT-1 transporters) and $0.3 \mathrm{~mm}$ t-PDC (a nonselective uptake antagonist; $n=8$ ) (Fig. $1 F$ ) or after replacement of the extracellular sodium by lithium, which inhibits the sodium-dependent glutamate uptake $(2.4 \pm 1.2 \%$ from control; $n=6$; $p<0.001$; data not shown). In contrast, the amplitude of the $\mathrm{Ca}^{2+}$ elevations was not significantly affected by t-PDC plus DHK $(n=8)$

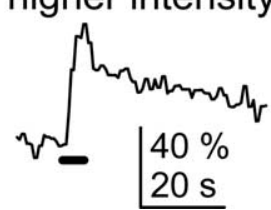

(Fig. 1G). Therefore, the astrocytic inward current was mediated by activation of electrogenic glutamate transporters.

We then pharmacologically investigated the mechanisms underlying the astrocytic $\mathrm{Ca}^{2+}$ elevations. The $\mathrm{SC}$-induced astrocytic $\mathrm{Ca}^{2+}$ increases were abolished by GluR antagonists $(20 \mu \mathrm{M}$ CNQX plus $50 \mu \mathrm{M}$ AP-5 plus $0.8 \mathrm{~mm} \mathrm{MCPG;} n=8$ ) (Fig. 1E,G). Although the expression of ionotropic GluRs has been reported in glial cells including astrocytes (Shelton and McCarthy, 1999; Bergles et al., 2000), $\mathrm{Ca}^{2+}$-permeable GluRs did not contribute substantially to the $\mathrm{Ca}^{2+}$ elevations because they were unaffected by ionotropic GluR antagonists $(20 \mu \mathrm{M}$ CNQX plus 50 $\mu \mathrm{M}$ AP-5; $69.5 \pm 11.1 \% ; n=4 ; p=0.07$; data not shown) but were abolished by the metabotropic GluR (mGluR) antagonist $\operatorname{MCPG}(0.8 \mathrm{~mm} ; n=8)$, indicating that the $\mathrm{SC}$-evoked $\mathrm{Ca}^{2+}$ signal was essentially mediated by mGluRs (Fig. 2B). Consistent with these findings, thapsigargin $(1 \mu \mathrm{M})$, a $\mathrm{Ca}^{2+}$-ATPase inhibitor that depletes internal $\mathrm{Ca}^{2+}$ stores, abolished the synaptically induced $\mathrm{Ca}^{2+}$ elevations without affecting the amplitude of the inward current, which reflects the amount of synaptically released glutamate (Bergles and Jahr, 1997; Bergles et al., 1999) $(n=5)$ (Fig. $1 F, G$ ). Therefore, the astrocyte $\mathrm{Ca}^{2+}$ increase was attributable to $\mathrm{Ca}^{2+}$ mobilization from internal stores, which further supports that the $\mathrm{Ca}^{2+}$ signal was mediated by G-protein-coupled mGluRs.

Astrocytes discriminate the activity of different synaptic terminals belonging to different axon pathways

Although we previously demonstrated that stratum oriens astrocytes responded with $\mathrm{Ca}^{2+}$ elevations to ACh but not glutamate released from the alveus synaptic terminals (Araque et al., 2002), present results show that astrocytes can be activated by glutamate released from SCs, suggesting that astrocytes discriminate the activity of different glutamatergic synapses and respond selectively to the activity of either pathway. To further address this issue, we stimulated both pathways while recording from single astrocytes. SC-evoked $\mathrm{Ca}^{2+}$ elevations were insensitive to the muscarinic cholinergic receptor $(\mathrm{mAChR})$ antagonist atropine $(50 \mu \mathrm{M})$ but abolished by MCPG (Fig. 2 B, D). Conversely, atropine, but not MCPG, prevented alveus-evoked $\mathrm{Ca}^{2+}$ elevations (Fig. $2 \mathrm{D}) . \mathrm{Ca}^{2+}$ variations were prevented in the presence of both atropine and MCPG. Therefore, $\mathrm{Ca}^{2+}$ elevations evoked by either alveus or SC stimulation were selectively mediated by $\mathrm{mAChRs}$ and mGluRs, respectively. These results indicate that astrocytic GluRs were activated by glutamate released from SC 
terminals but not by glutamate released from alveus axons, which, however, reached the astrocytic membrane as assessed by the recorded glutamate transporter-mediated currents (Fig. 2A) (cf. Araque et al., 2002).

Together, these results indicate that astrocytes specifically respond to different axon pathways that release different neurotransmitters (i.e., ACh and glutamate). Furthermore, because they display $\mathrm{Ca}^{2+}$ elevations evoked by glutamate released from SCs but not from glutamatergic axons in the alveus, astrocytes discriminate the activity of different synaptic terminals belonging to different axon pathways.

\section{Astrocyte $\mathrm{Ca}^{2+}$ signal is modulated by interaction of neurotransmitters released by different synaptic inputs}

A remarkable feature of neurons is their ability to modulate their cellular excitability by the interaction of different neurotransmitters (McCormick, 1998). Because stratum oriens astrocytes responded with $\mathrm{Ca}^{2+}$ elevations to the activity of cholinergic and glutamatergic axons, we investigated whether the astrocytic $\mathrm{Ca}^{2+}$ signal could also be modulated by the interaction of both pathways. We compared the astrocytic responses evoked by either independent or simultaneous stimulation of the alveus and SC. We reasoned that if there was no interaction, the astrocytic responses elicited simultaneously would be the linear summation of the responses evoked independently. The interaction was quantified from the O/E ratio: the ratio between the observed response evoked by simultaneous stimulation and the expected response (i.e., the summation of the responses evoked by independent stimulation). Although the inward current evoked by simultaneous stimulation was not significantly different from the expected response, the $\mathrm{Ca}^{2+}$ elevation induced by concurrent stimulation was different from the expected one (Fig. $2 A-C$ ). In 10 of 77 cases, the $\mathrm{Ca}^{2+}$ signal evoked by simultaneous stimulation was higher than the summation of the $\mathrm{Ca}^{2+}$ elevations elicited independently, but in most cases (60 of 77 cells), the $\mathrm{Ca}^{2+}$ signal was lower (i.e., $\mathrm{O} / \mathrm{E}<100 \%$ ). On average, during simultaneous stimulation, the synaptically evoked $\mathrm{Ca}^{2+}$ signal was reduced $47.8 \pm 5.6 \%(n=77)$ (Fig. $2 C)$.

In the presence of atropine, which abolished the alveusinduced $\mathrm{Ca}^{2+}$ elevation without affecting the SC-induced response, the amplitude of the observed and expected $\mathrm{Ca}^{2+}$ signal was similar (Fig. 2B,D). Likewise, in the presence of MCPG, which prevented the $\mathrm{SC}$-induced $\mathrm{Ca}^{2+}$ elevation without affecting the alveus-evoked response, the observed and expected responses were not significantly different (Fig. 2D). Therefore, the activation of both $\mathrm{mAChRs}$ and mGluRs were required to modulate the astrocytic $\mathrm{Ca}^{2+}$.

The $\mathrm{Ca}^{2+}$ signal modulation cannot be accounted for by the saturation of the machinery of $\mathrm{Ca}^{2+}$ release from internal stores or their partial depletion, because increasing the stimulation intensity (which recruits more axons) increased the $\mathrm{Ca}^{2+}$ levels beyond the amplitude of the expected response $(n=4)$ (Fig. $2 E$ ). Furthermore, the $\mathrm{O} / \mathrm{E}$ ratio did not correlate with the amplitude of the independently evoked responses $\left(r^{2}=0.01\right.$ for both SCand alveus-induced $\mathrm{Ca}^{2+}$ elevations; data not shown). Moreover, some astrocytes only showed detectable $\mathrm{Ca}^{2+}$ elevations to either the alveus ( 11 of 77 cells) or the SC ( 24 of 77 cells) stimulation but not to stimulation of the other pathway, probably because $\mathrm{Ca}^{2+}$ elevations were below our detection level. Nevertheless, these astrocytes also displayed $\mathrm{Ca}^{2+}$ modulation after simultaneous stimulation (33 of 35 astrocytes), which suggests that practically all of the astrocytes responded to both pathways. More impor-

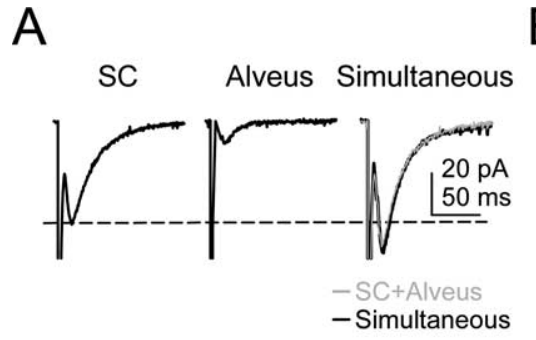

B
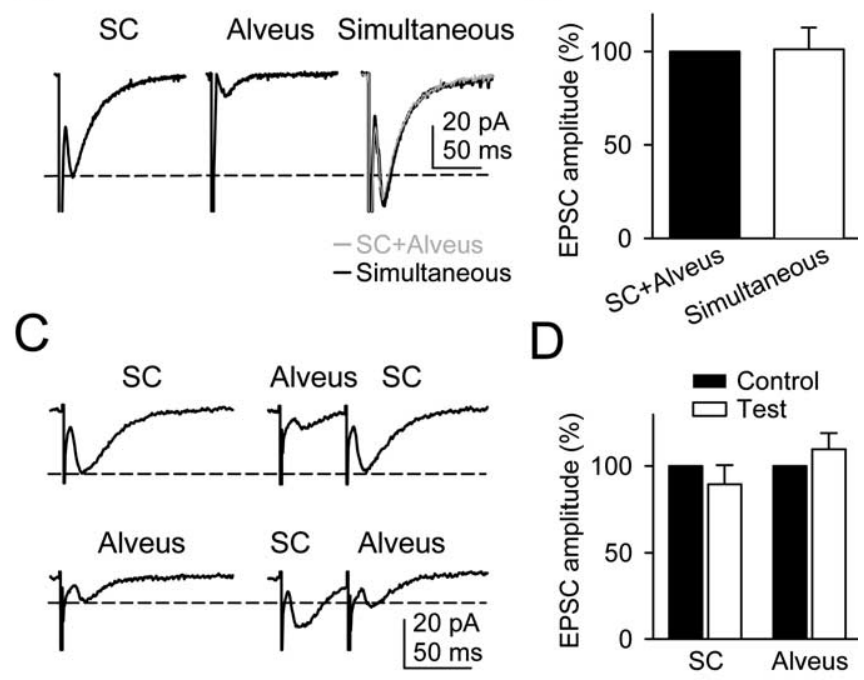

$E$
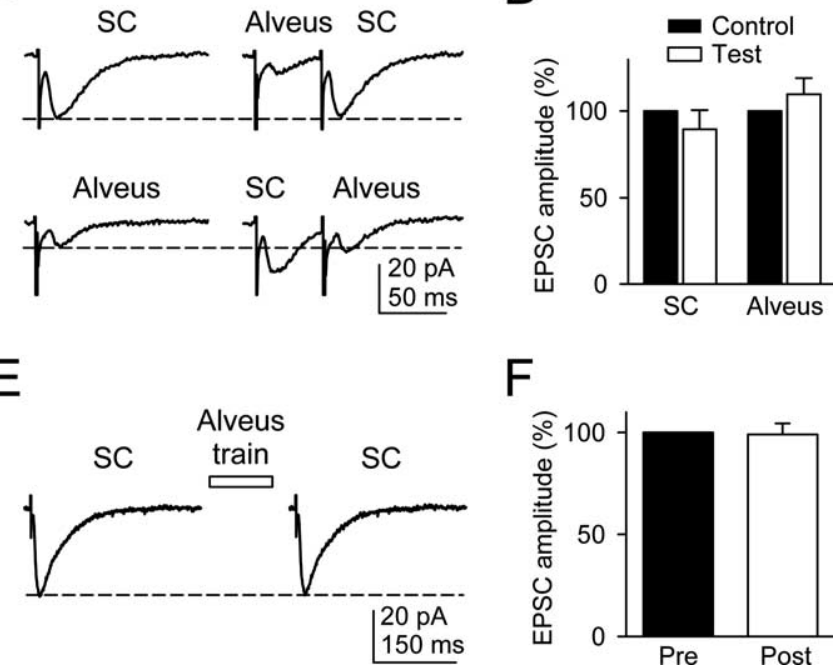

$\mathrm{F}$

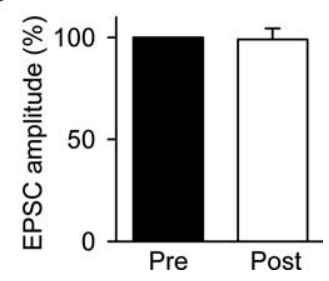

Figure 3. EPSCS evoked by $S C$ and alveus stimulation were unaffected by the activity of either pathway. $\boldsymbol{A}$, Mean EPSCS $(n=20)$ elicited by the SC, alveus, and simultaneous stimulation of both pathways. $\boldsymbol{B}$, Averaged EPSC amplitudes evoked by simultaneous stimulation of the $S C$ and alveus relative to the linear summation of EPSCs evoked independently (SC plus alveus) ( $n=5$ neurons). C, Mean EPSCs $(n=20)$ evoked by the SC and alveus (top and bottom traces, respectively) in controls and after alveus or $S C$ stimulation, respectively. $\boldsymbol{D}$, Averaged relative EPSC amplitudes evoked by the SC and alveus in controls and after stimulation of the alveus and SC (Test), respectively ( $n=5$ cells). $\boldsymbol{E}$, Mean SC-evoked EPSCs $(n=20)$ before and after stimulation of the alveus with a single train $(30 \mathrm{~Hz}, 5 \mathrm{~s})$. $\boldsymbol{F}$, Averaged relative SC-evoked EPSC amplitudes before (Pre) and after (Post) alveus stimulation ( $n=8$ neurons).

tant, these results support the notion that the $\mathrm{Ca}^{2+}$ signal modulation was not attributable to the saturation of the $\mathrm{Ca}^{2+}$ source.

The reduction in the $\mathrm{Ca}^{2+}$ signal observed during simultaneous stimulation was unlikely the result of a decrease in neurotransmitter release, because the observed inward current amplitude, which reflects the amount of released glutamate (Mennerick et al., 1996; Bergles and Jahr, 1997; Bergles et al., 1999), was similar to the expected current (Fig. 2A-C). To further address this possibility, we used a more sensitive approach to detect changes in transmitter release by recording SC- and alveus-evoked synaptic currents in CA1 pyramidal neurons. SC and alveus stimulation evoked EPSCs that were abolished by 20 $\mu \mathrm{M}$ CNQX ( $n=3$; data not shown). The synaptic currents evoked by simultaneous stimulation were not significantly different from the summation of EPSCs evoked independently $(n=5)$ (Fig. $3 A, B)$. Furthermore, the SC-evoked EPSC amplitude was not affected when preceded by the alveus-evoked EPSC ( $n=5$ neurons) (Fig. 3C,D). Likewise, the alveus-evoked EPSC amplitude was unaffected by previous stimulation of SCs $(n=5)$ (Fig. $3 C, D)$. Together, the summation and the absence of crossregulation of EPSCs indicate that different sets of synapses were activated by each stimulating electrode and that the amount of transmitter released by either the alveus or SC was not modified by the stimulation of the other pathway. 
A

B

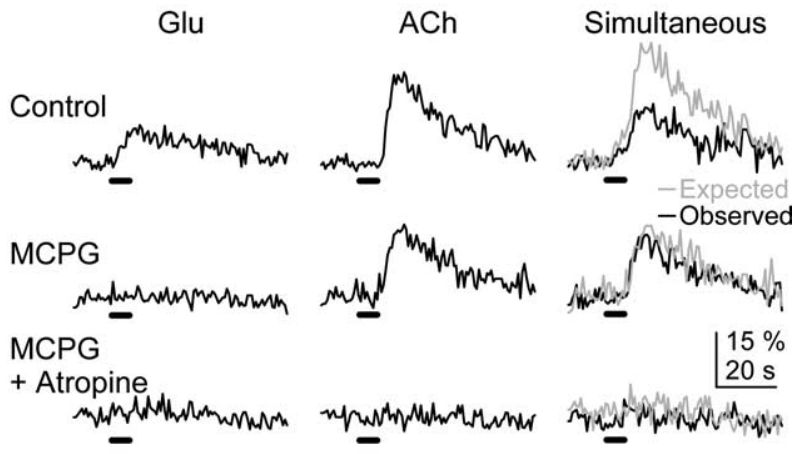

C

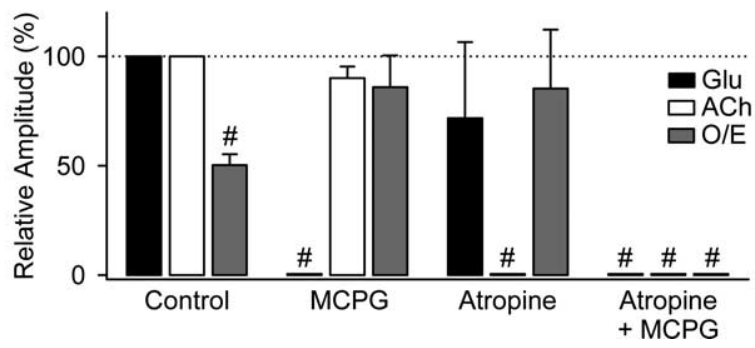

Figure 4. $\quad \mathrm{Ca}^{2+}$ signal modulation depended on astrocytic intrinsic properties. A, Schematic drawing showing an astrocyte whole cell filled with fluo-3 and a double-barreled pipette filled with $\mathrm{ACh}$ and $\mathrm{Glu}$ and $\mathrm{Ca}^{2+}$ elevations evoked by simultaneous application of Glu and $\mathrm{ACh}$ relative to the linear summation of responses elicited independently (control) $(n=15)$. $\boldsymbol{B}$, Astrocytic $\mathrm{Ca}^{2+}$ levels evoked by independent or simultaneous ionophoresis of $\mathrm{Glu}$ and $\mathrm{ACh}$ in controls, in $0.8 \mathrm{~mm} \mathrm{MCPG}$, and after the addition of $50 \mu \mathrm{m}$ atropine. C, Relative amplitude of the $\mathrm{Ca}^{2+}$ elevations evoked by ionophoresis of Glu and ACh in control conditions and in $0.8 \mathrm{~mm}$ $\mathrm{MCPG}, 50 \mu \mathrm{m}$ atropine, or both. Responses evoked by simultaneous stimulation were quantified from the $0 / E$ ratio (see Results). $n \geq 5$ for each bar. Significant differences were established by the Student's $t$ test at ${ }^{\#} p<0.001$.

Because ACh may modulate neurotransmitter release, and repetitive long-lasting stimulation of cholinergic afferents induces a slowly developing inhibition of glutamate release from SC terminals (Fernández de Sevilla and Buño, 2003), we investigated whether SC-evoked EPSCs were modulated by stimulation of cholinergic axons in the alveus with a single train of stimuli (30 $\mathrm{Hz}, 5 \mathrm{~s}$ ) (i.e., the same parameters used to monitor astrocyte $\mathrm{Ca}^{2+}$ signal modulation). The mean SC-evoked EPSC amplitude was not significantly different before and after alveus stimulation $(n=8)$ (Fig. $3 E, F)$. Together, the analysis of synaptic currents indicates that possible neural network interactions could not account for the modulation of the astrocyte $\mathrm{Ca}^{2+}$ signal.

$\mathrm{Ca}^{2+}$ signal modulation depends on cellular intrinsic properties of astrocytes

Because the $\mathrm{Ca}^{2+}$ signal modulation required receptor activation and did not depend on neural network interaction, we predicted that a similar regulation would be elicited by direct application of transmitters. To test this hypothesis, glutamate and ACh were
A
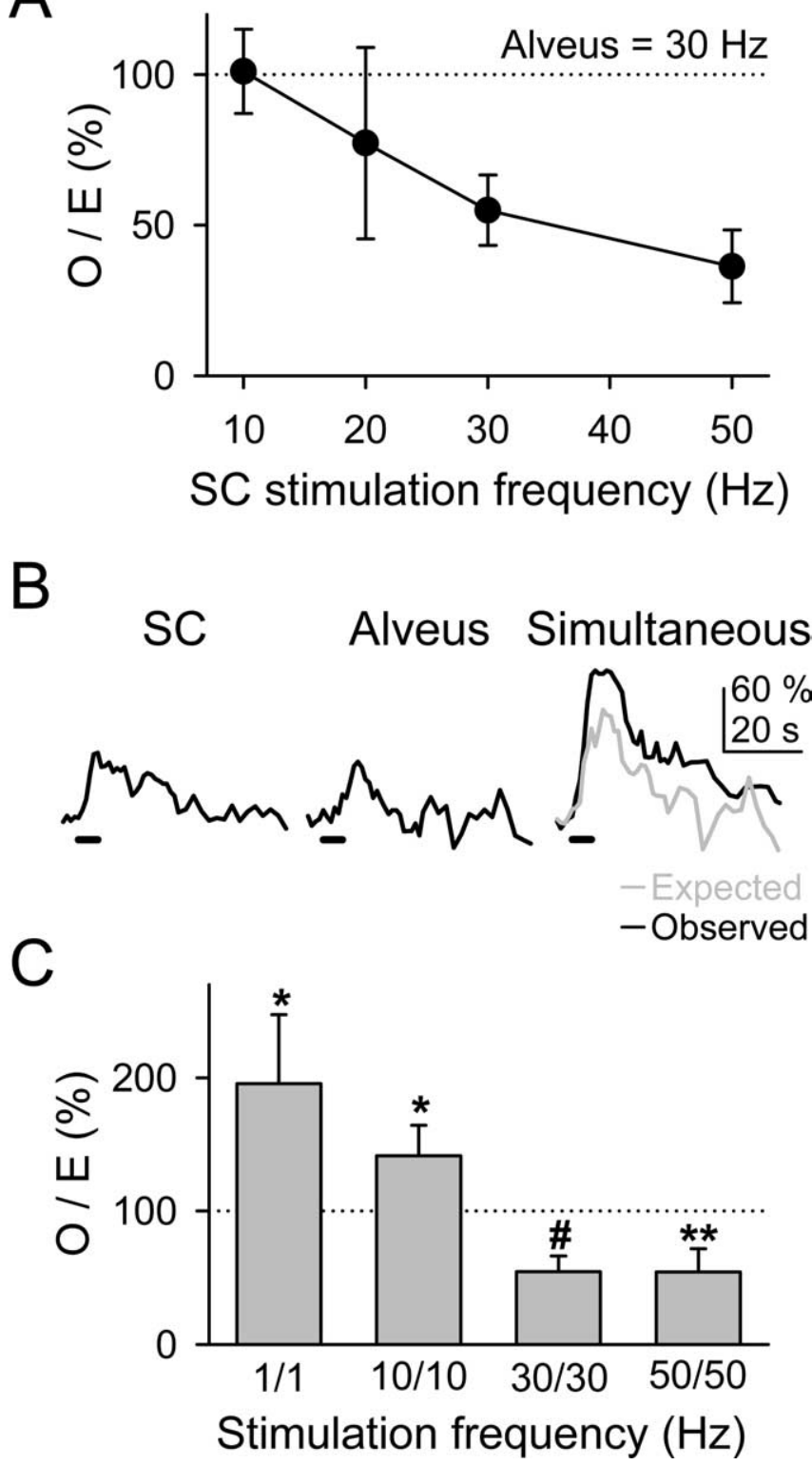

Figure 5. Modulation of the astrocytic $\mathrm{Ca}^{2+}$ signal depended on the synaptic activity level. $A$, Plot of the 0/E ratio versus the $S C$ stimulation frequency. Observed and expected $\mathrm{Ca}^{2+}$ elevations corresponded with the simultaneous responses and with the linear summation of the independent responses, respectively, evoked by stimulation of the alveus at $30 \mathrm{~Hz}$ and of the SC at variable frequencies ( $n \geq 9$ for each value). $\boldsymbol{B}, \mathrm{Ca}^{2+}$ elevations evoked by independent or simultaneous stimulation of the $\mathrm{SC}$ and alveus with trains of stimuli at $10 \mathrm{~Hz}$ for $5 \mathrm{~s} . \mathrm{C}, 0 / \mathrm{E}$ ratio obtained by varying concurrently the stimulation frequencies of the $S C$ and alveus at $1,10,30$ and $50 \mathrm{~Hz}$ ( $n \geq 10$ for each bar). Significant differences from control values were established by the Student's $t$ test at ${ }^{*} p<0.05,{ }^{* *} p<0.01$, and ${ }^{\#} p<0.001$.

microionophoretically applied through a double-barreled micropipette positioned close to the recorded astrocyte. Application of either glutamate or ACh for $5 \mathrm{~s}$ evoked $\mathrm{Ca}^{2+}$ elevations that were sensitive to $0.8 \mathrm{~mm} \mathrm{MCPG}(n=6)$ and $50 \mu \mathrm{M}$ atropine $(n=$ $5)$, respectively. $\mathrm{Ca}^{2+}$ elevations evoked by simultaneous ionophoresis of both transmitters were significantly smaller than the expected responses $(n=15)$ (Fig. $4 A, B)$. The $\mathrm{Ca}^{2+}$ signal modulation disappeared when either mGluRs or mAChRs were antagonized (Fig. $4 B, C$ ). These results, which strongly resemble those obtained by synaptic stimulation, confirm that the $\mathrm{Ca}^{2+}$ signal modulation was not attributable to neuronal network reg- 
A

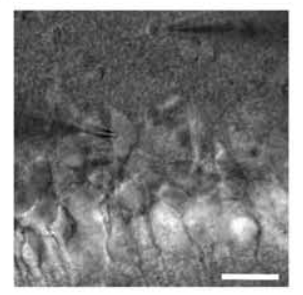

B

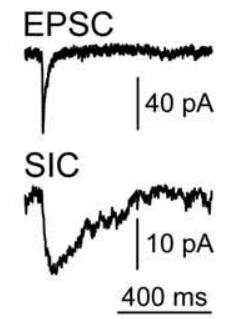

C

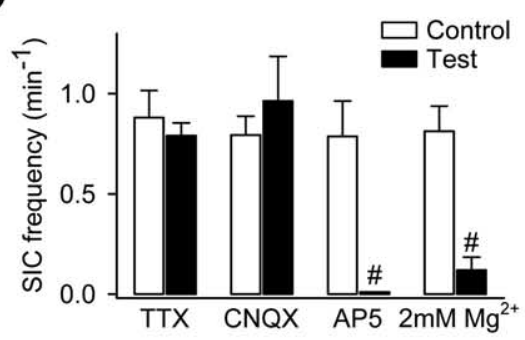

D

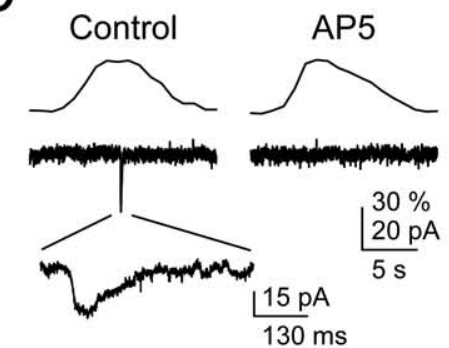

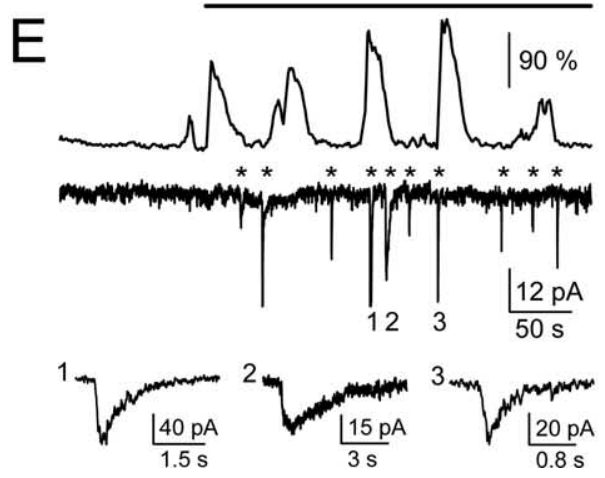

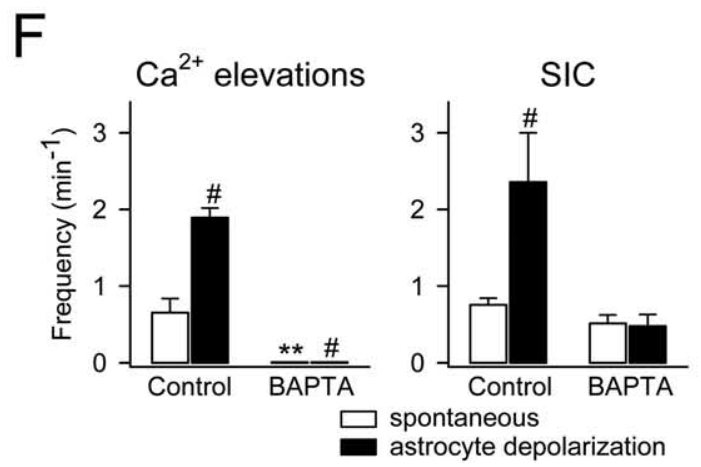

G

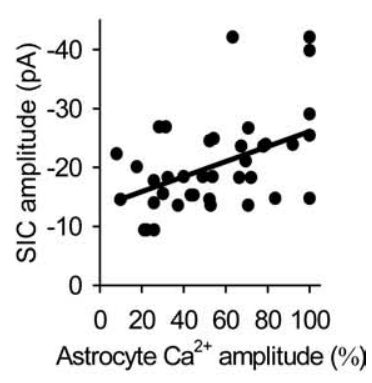

Figure 6. $\quad \mathrm{Ca}^{2+}$ elevations in astrocytes evoked NMDAR-mediated SICs in pyramidal neurons. $A$, Infrared differential interference contrast image. Note the recording pipettes to the right of the astrocyte in the stratum oriens (top) and to the left of the neuron in the pyramidal layer (bottom). Scale bar, $25 \mu \mathrm{m}$. B, Representative EPSC and SIC recorded from a CA1 pyramidal neuron. Note their

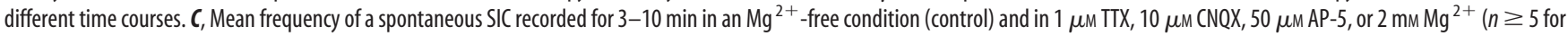
each bar). D, Representative $\mathrm{Ca}^{2+}$ levels (top traces) and whole-cell currents (bottom traces) simultaneously recorded in astrocytes and neurons, respectively. As in $69.2 \pm 4.8 \%$ of the cases, the neuronal SIC (expanded in the bottom trace) occurred in parallel with a $\mathrm{Ca}^{2+}$ elevation in an adjacent astrocyte. A concentration of $50 \mu \mathrm{M} \mathrm{AP-5}$ abolished the SIC but did not affect astrocytic Ca ${ }^{2+}$ elevations $(n=5) . \boldsymbol{E}$, Representative astrocyte $\mathrm{Ca}^{2+}$ signal and neuronal current obtained from paired whole-cell recordings in controls and after astrocyte stimulation (top horizontal bar) with 180 $\mathrm{mV}$ depolarizing pulses (duration, $100-500 \mathrm{~ms}$ ) delivered every $1 \mathrm{~s}$. The asterisks indicate neuronal SICs. Some SICs have been truncated, and three are expanded in the bottom traces. Note the frequency increase of both astrocyte $\mathrm{Ca}^{2+}$ elevation and neuronal SIC after astrocyte stimulation. $F$, Mean frequency of astrocytic $\mathrm{Ca}^{2+}$ elevations and neuronal SICs recorded spontaneously ( $\square$ ) or during astrocytic depolarization $(\square)$ in control conditions or in the presence of $20 \mathrm{~mm}$ BAPTA in the recording pipette of the astrocyte $(n=6)$. Significant differences from control values were established by the Student's $t$ test at ${ }^{* *} p<0.01$ and ${ }^{\#} p<0.001$. G, Plot of the neuronal SIC amplitude versus the fluorescence amplitude recorded in the soma of the astrocyte $(n=39$ from 6 pairs of cells). The fluorescence amplitude was normalized to the highest amplitude for each astrocyte. Only SICs that coincided with $\mathrm{Ca}^{2+}$ elevations were considered. Points were fitted to a linear regression (straight line; $r^{2}=0.20$ ).

ulation, but it rather depended on cellular intrinsic properties of astrocytes.

\section{Modulation of astrocytic $\mathrm{Ca}^{2+}$ is regulated by the synaptic activity level}

We investigated the relationship between the degree of synaptic activity and the modulation of the astrocyte $\mathrm{Ca}^{2+}$ signal. We first analyzed the $\mathrm{O} / \mathrm{E}$ ratio at different SC stimulation frequencies (from 10 to $50 \mathrm{~Hz} ; 5 \mathrm{~s}$ duration trains) while maintaining the alveus stimulation frequency at $30 \mathrm{~Hz}$. Figure $5 \mathrm{~A}$ shows that at these frequencies, the $\mathrm{O} / \mathrm{E}$ ratio decreased as the $\mathrm{SC}$ stimulation frequency increased (i.e., the relative depression of the $\mathrm{Ca}^{2+}$ signal increased as the synaptic activity increased). Because the alveus stimulation used was relatively high $(30 \mathrm{~Hz})$, to study the effects of lower frequencies, we used a different stimulation paradigm by changing concomitantly the frequency of both synaptic pathways (Fig. $5 B, C$ ) and analyzed the respective $\mathrm{O} / \mathrm{E}$ ratios. Whereas at high frequencies $(30$ and $50 \mathrm{~Hz})$ the observed $\mathrm{Ca}^{2+}$ elevations were significantly smaller than the expected responses, at low frequencies $(1$ and $10 \mathrm{~Hz}$ ), the observed responses were significantly higher, indicating the existence of a bidirectional control of the astrocyte $\mathrm{Ca}^{2+}$ signal by synaptic activity.

\section{$\mathrm{Ca}^{2+}$ elevations in astrocytes evoke NMDAR-mediated} currents in neurons

To investigate the electrophysiological consequences of the astrocyte $\mathrm{Ca}^{2+}$ elevations on neuronal excitability, we performed paired whole-cell recordings of astrocytes and CA1 pyramidal neurons, while simultaneously monitoring astrocyte $\mathrm{Ca}^{2+}$ levels. Because nerve stimulation could directly induce neuronal responses that could be erroneously identified as astrocytemediated events, we rather analyzed the neuronal responses to astrocyte $\mathrm{Ca}^{2+}$ elevations that occurred spontaneously or after direct depolarizing stimulation (Fig. 6A).

In the absence of extracellular $\mathrm{Mg}^{2+}$, to minimize NMDAR blockade, neurons displayed spontaneous EPSCs $(-39.8 \pm 3.9$ $\mathrm{pA} ; n=35)$ as well as low-frequency $\left(0.79 \pm 0.09 \mathrm{~min}^{-1} ; n=17\right)$ SICs $(-18.3 \pm 1.4 \mathrm{pA} ; n=35)$, which could be distinguished by their onset and decay time courses (EPSCs: $\tau_{\text {on }}=2.7 \pm 0.5 \mathrm{~ms}$, fast $\tau_{\text {off }}=3.7 \pm 0.6 \mathrm{~ms}$, slow $\tau_{\text {off }}=30.6 \pm 3.8 \mathrm{~ms}$; SIC: $\tau_{\text {on }}=$ $13.9 \pm 1.7 \mathrm{~ms}, \tau_{\text {off }}=72.5 \pm 11.1 \mathrm{~ms} ; n=35$ ) (Fig. $6 B$ ). The SIC frequency was insensitive to TTX $(n=6)$, indicating that it was not mediated by action potential-evoked transmitter release. Furthermore, in this condition, SICs and miniature EPSCs could also be distinguished by their different kinetic properties (10$90 \%$ rise time, $27.9 \pm 8.5$ and $6.0 \pm 1.0 \mathrm{~ms}$, respectively; $10-90 \%$ decay time, $193.2 \pm 92.9$ and $24.5 \pm 3.2 \mathrm{~ms}$, respectively; $n=20$ ).

The SIC frequency was unaffected by CNQX $(10 \mu \mathrm{M} ; n=5)$, but it was reduced in the presence of $2 \mathrm{mM} \mathrm{Mg}^{2+}$ (from $0.81 \pm$ 0.12 in controls to $0.12 \pm 0.06 \mathrm{~min}^{-1} ; n=6$ ) and abolished by AP-5 (50 $\mu \mathrm{M} ; n=5$ ), suggesting that the SIC was selectively mediated by NMDARs (Fig. 6C,D). Unexpectedly, the mean SIC amplitude was not significantly different in controls and in $2 \mathrm{mM}$ $\mathrm{Mg}^{2+}(-22.8 \pm 3.2$ and $-17.0 \pm 1.9 \mathrm{pA}$, respectively; $n=17$; 
$p>0.05$ ), which is probably because of the fact that lowamplitude SICs in $2 \mathrm{~mm} \mathrm{Mg}^{2+}$ were below the detection level.

Astrocytes from different brain areas exhibit spontaneous intrinsic $\mathrm{Ca}^{2+}$ elevations that may stimulate the release of glutamate (Pasti et al., 1997; Parri et al., 2001; Aguado et al., 2002; Nett et al., 2002). We investigated the relationship between the appearance of neuronal SIC and the intrinsic astrocytic $\mathrm{Ca}^{2+}$ elevations, which were simultaneously monitored in several astrocytes at a time (usually three to six astrocytes close to the recorded neuron) by previous incubation of the slices with fluo-3 AM. Most SICs (69.2 $\pm 4.8 \% ; n=92$ from eight pair cells) were observed during $\mathrm{Ca}^{2+}$ elevations in an adjacent astrocyte (Fig. $6 D, E)$. The absence of total parallelism between the astrocytic $\mathrm{Ca}^{2+}$ and the neuronal SIC is not unexpected, because it is likely that a fraction of the astrocytes experiencing $\mathrm{Ca}^{2+}$ elevations were not monitored (e.g., because they were out of focus or inefficiently loaded with fluo-3). Because stimulation of astrocytes with trains of depolarizing pulses induces $\mathrm{Ca}^{2+}$ elevations and oscillations (Kang et al., 1998; Zonta et al., 2003), to investigate whether the SIC frequency was modulated by the astrocyte $\mathrm{Ca}^{2+}$, we directly depolarized the astrocyte through the recording pipette while simultaneously recording its $\mathrm{Ca}^{2+}$ levels as well as the neuronal currents. Astrocyte depolarization increased the frequency of occurrence of both astrocyte $\mathrm{Ca}^{2+}$ elevations and SIC $(n=8)$ (Fig. 6E,F). AP-5 abolished the SIC frequency increase without affecting the frequency increase of the astrocyte $\mathrm{Ca}^{2+}$ elevations $(n=5)$ (Fig. $6 D)$, supporting the idea that glutamate released from astrocytes evoked the neuronal SIC. To test whether the astrocyte $\mathrm{Ca}^{2+}$ elevations were necessary to induce the SIC, 20 mM BAPTA was included in the recording pipette of the astrocyte. In this situation, no $\mathrm{Ca}^{2+}$ variations were observed in the astrocyte, either in resting or stimulated conditions, and astrocyte stimulation did not increase the SIC frequency $(n=6)$ (Fig. 6F).

To confirm that $\mathrm{Ca}^{2+}$ elevations in astrocytes caused the neuronal SIC, we synchronized the elevation of $\mathrm{Ca}^{2+}$ levels in a group of astrocytes by ionophoretical application of ATP (Charles and Giaume, 2002; Nedergaard et al., 2003; Newman, 2003). In the presence of TTX, ATP increased astrocyte $\mathrm{Ca}^{2+}$ levels and evoked single or multiple SICs in neurons $(-77.7 \pm 1.3$ pA; $n=40$ from five slices) (Fig. $7 A, B$ ). Although SICs were abolished by AP-5, the ATP-evoked $\mathrm{Ca}^{2+}$ elevations were unaffected ( $n=5$ slices) (Fig. $7 B, C$ ), supporting the idea that astrocyte $\mathrm{Ca}^{2+}$ elevations stimulate glutamate release that evoke NMDAR-mediated neuronal currents. Despite the sustained $\mathrm{Ca}^{2+}$ increases, the SIC tended to occur during the rising phase of the astrocyte $\mathrm{Ca}^{2+}$ elevations (Fig. 7D), suggesting that the glutamate release mechanism acts as a detector of intracellular $\mathrm{Ca}^{2+}$ variations. Together, these results indicate that $\mathrm{Ca}^{2+}$ elevations in astrocytes evoke NMDAR-mediated SICs in CA1 pyramidal neurons.

We investigated whether the amplitude of the glutamatemediated SIC depended on the amplitude of the $\mathrm{Ca}^{2+}$ signal detected at the astrocytic soma. We selected pairs of simultaneous neuronal SICs and astrocytic $\mathrm{Ca}^{2+}$ elevations (occurring spontaneously or after astrocyte depolarization) (i.e., when the recorded SIC occurred during the astrocyte $\mathrm{Ca}^{2+}$ elevation) and plotted the SIC amplitude against the relative fluorescence amplitude recorded in the soma of the astrocyte (Fig. 6G). The SIC amplitude was only weakly correlated with the astrocytic $\mathrm{Ca}^{2+}$ at the soma $\left(r^{2}=0.20 ; n=39\right.$ from six cell pairs), which would favor the idea that glutamate-mediated astrocyte-to-neuron signaling is a highly localized event occurring on restricted cellular regions
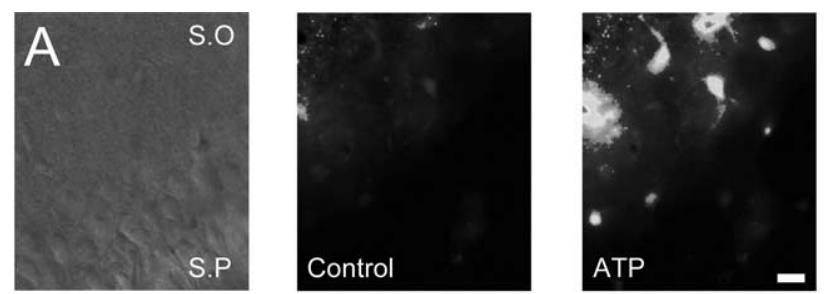

B
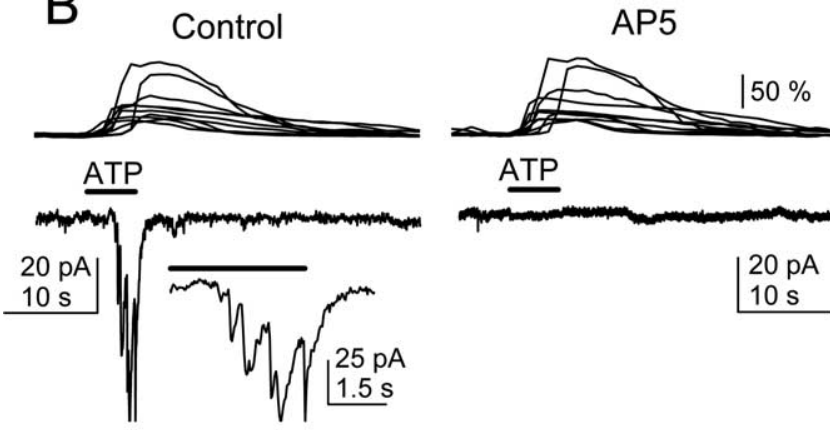

ATP
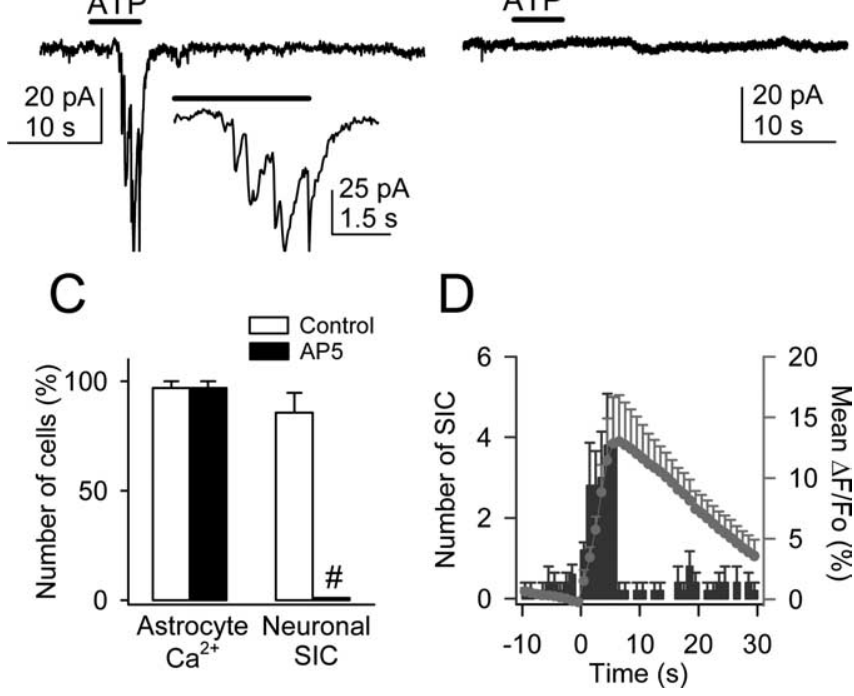

Figure 7. ATP-induced $\mathrm{Ca}^{2+}$ elevations in astrocytes evoked NMDAR-mediated SICs in pyramidal neurons. $A$, Infrared differential interference contrast image and pseudocolor images representing fluorescence intensities of a fluo-3-filled slice before and after ionophoretical application of ATP for $5 \mathrm{~s}$. Note the lower relative fluorescence at the pyramidal layer. S.0, Stratum oriens; S.P, stratum pyramidale. Scale bar, $30 \mu \mathrm{m}$. B, Astrocyte $\mathrm{Ca}^{2+}$ levels (top traces) and whole-cell neuronal currents (bottom traces) during ionophoretical application of ATP (horizontal bar) in TTX and without $\mathrm{Mg}^{2+}$ (control and after perfusion with $50 \mu \mathrm{M} \mathrm{AP-5)}$. Inset, Expanded current trace illustrating the multiple NMDAR-mediated SICs. C, Relative number of astrocytes and neurons that showed $\mathrm{Ca}^{2+}$ elevations and SICS, respectively, after application of ATP in controls and after perfusion with AP- 5 ( $n=35$ astrocytes and 5 neurons from 5 slices). Significant differences were established by the Student's $t$ test at ${ }^{\#} p<0.001$. $\boldsymbol{D}$, Mean number of neuronal SICs (blue bars) and averaged astrocyte $\mathrm{Ca}^{2+}$ elevations (red circles) versus time ( $n=5$ slices). Time 0 corresponds to the beginning of the ATP application ( $5 \mathrm{~s}$ ).

called microdomains (Pasti et al., 1997; Grosche et al., 1999; Carmignoto, 2000; Araque et al., 2002; Haydon and Araque, 2002; Nett et al., 2002; Fiacco and McCarthy, 2004). We therefore investigated whether the $\mathrm{Ca}^{2+}$ signal modulation was also present at astrocytic restricted regions (Fig. 8). We were not able to identify functional microdomains [i.e., subcellular regions that respond independently to synaptic activity (Grosche et al., 1999)] because under high-frequency stimulation, which was required to depress the astrocytic response, the $\mathrm{Ca}^{2+}$ signal propagated throughout the cell (see below). Nevertheless, we measured the fluorescence in restricted regions of the processes with the defined magnitude of microdomains in Bergmann glia (i.e., 20-50 $\mu \mathrm{m}^{2}$ ), and we analyzed a single region per process (Fig. $8 \mathrm{C}$ ). Most subcellular regions (20 of 24 , from 14 astrocytes) displayed $\mathrm{Ca}^{2+}$ signal modulation by simultaneous activation of the SC and alveus as indicated by the corresponding $\mathrm{O} / \mathrm{E}$ ratio. Indeed, although the simultaneously evoked $\mathrm{Ca}^{2+}$ signal was reduced in 17 
of 24 restricted regions, it was potentiated or unchanged in three and four cases, respectively, indicating that the $\mathrm{Ca}^{2+}$ signal modulation is not only manifested in the soma but also in the processes.

We studied the effects of the $\mathrm{Ca}^{2+}$ signal modulation on the intracellular $\mathrm{Ca}^{2+}$ signal propagation. In some cases [ 12 of 14 cells $(86 \%)]$, astrocytic responses evoked by afferent stimulation could be observed as a $\mathrm{Ca}^{2+}$ elevation that initiated in a process and eventually propagated to the soma and other processes (cf. Porter and McCarthy, 1996; Pasti et al., 1997; Kang et al., 1998; Grosche et al., 1999; Parri et al., 2001). In 10 of those 14 cells (i.e., 71\%), the simultaneous stimulation controlled the propagation of the synaptically evoked $\mathrm{Ca}^{2+}$ signal. Indeed, Figure $8, D$ and $E$, shows an example in which alveus stimulation evoked a $\mathrm{Ca}^{2+}$ increase in region 1 that later propagated with delay to the soma and region 3. After simultaneous stimulation, the observed $\mathrm{Ca}^{2+}$ signal was not only relatively depressed from the expected values, but it was also confined to region 1 , failing to propagate to the soma and region 3 , indicating that simultaneous activity of different synapses not only modulated the amplitude of the $\mathrm{Ca}^{2+}$ signal in astrocytic processes but also controlled its intracellular propagation.

Together, these results support the idea that the astrocytic subcellular microdomains are the elementary units underlying the reciprocal communication between astrocytes and neurons. Furthermore, the synaptic control of the intracellular $\mathrm{Ca}^{2+}$ propagation may have important consequences on brain function by regulating the spatial range of astrocyte influence on synaptic terminals.

\section{Discussion}

We investigated the properties of the synaptic-induced astrocytic $\mathrm{Ca}^{2+}$ signal, which is a key element in reciprocal astrocyte-neuron communication. We show that hippocampal astrocytes discriminate

\section{$\leftarrow$}

later propagated with delay to regions 2 and 3 . After simultaneous stimulation, $\mathrm{Ca}^{2+}$ increased in region 1 but failed to propagate to regions 2 and 3 . $\boldsymbol{F}$, Schematic drawing representing a hypothetical consequence of the $\mathrm{Ca}^{2+}$ signal modulation. Under independent high-frequency synaptic activity of either pathway (left), astrocyte $\mathrm{Ca}^{2+}$ elevations initiate in specific processes and then propagate to the soma and other processes, eventually leading to long-distance neuromodulation by $\mathrm{Ca}^{2+}$-dependent release of glutamate (arrows). However, simultaneous high-frequency synaptic activity prevents the intracellular propagation of the astrocyte $\mathrm{Ca}^{2+}$ signal and its long-distance neuromodulatory effects.
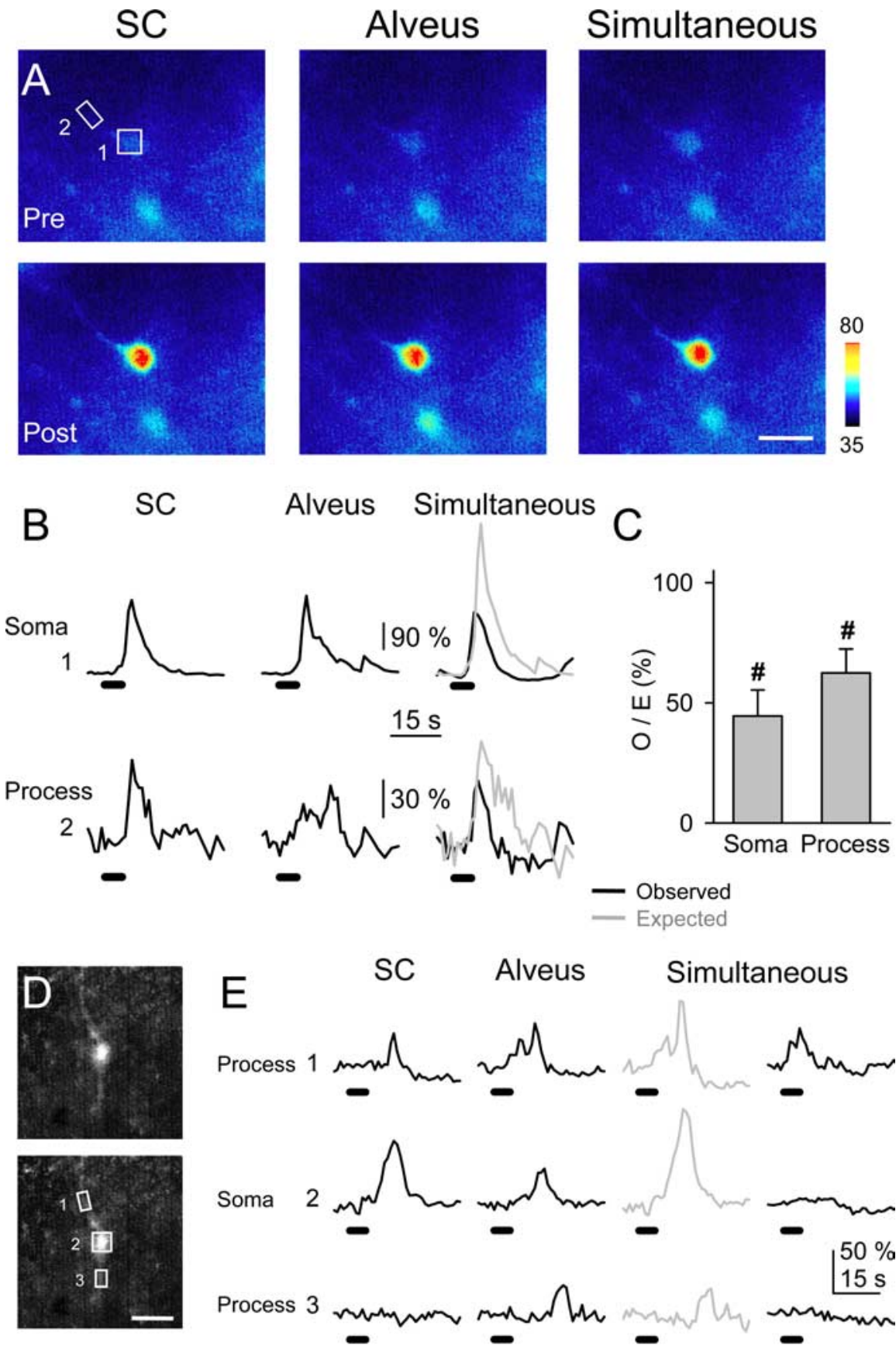

$\mathrm{F}$

$$
\begin{aligned}
& \text { Independent } \\
& \text { synaptic activity }
\end{aligned}
$$

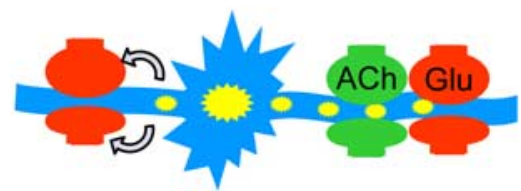

Figure 8. Astrocytic subcellular regions exhibited synaptic-mediated $\mathrm{Ca}^{2+}$ modulation. $\boldsymbol{A}$, Pseudocolor images representing fluorescence intensities of a fluo-3-filled astrocyte before (Pre) and $10 \mathrm{~s}$ after (Post) independent or simultaneous stimulation of the $\mathrm{SC}$ and alveus $(30 \mathrm{~Hz}, 5 \mathrm{~s})$. Scale bar, $15 \mu \mathrm{m}$. $\boldsymbol{B}$, Fluorescence intensity changes in the astrocytic soma and a restricted region of the astrocytic process marked with boxes 1 and 2, respectively, in $\boldsymbol{A}$. C, Ratio between observed and expected $\mathrm{Ca}^{2+}$ elevations in the soma $(n=14)$ and restricted regions at the processes $(n=24)$. Significant differences from control values were established by the Student's t test at ${ }^{\#} p<0.001$. D, Fluorescent images of a fluo-3-filled astrocyte. Scale bar, $15 \mu \mathrm{m}$. $\boldsymbol{E}$, Fluorescence intensity changes in restricted regions of two astrocytic processes (1 and 3) and soma (2) marked with boxes in $\boldsymbol{D}$, evoked by independent or simultaneous stimulation of the $\mathrm{SC}$ and alveus $(30 \mathrm{~Hz}, 5 \mathrm{~s})$. Note that after alveus stimulation, $\mathrm{Ca}^{2+}$ increased in region 1 and 
the activity of different synaptic terminals belonging to different axon pathways and that the astrocytic $\mathrm{Ca}^{2+}$ signal is bidirectionally modulated by interaction of different synaptic inputs because of cellular intrinsic properties, indicating that astrocytes integrate synaptic information.

Indeed, present data indicate that astrocytes discriminate between the activity of cholinergic and glutamatergic synapses as well as between the activity of different synapses belonging to different axon pathways (i.e., they distinguish between glutamate released from SC or alveus terminals). Furthermore, the astrocytic $\mathrm{Ca}^{2+}$ signal is modulated by the simultaneous activity of different synaptic inputs, and this regulation occurs in the absence of neural network interaction indicating that astrocytes display cellular intrinsic properties that control their excitability. We show that this modulation depended on the level of activity of synaptic terminals, being potentiated and depressed at low and high synaptic activity frequencies, respectively. This bidirectional modulation of the $\mathrm{Ca}^{2+}$ signal adds further complexity and degrees of freedom to the neuron-astrocyte communication and suggests that astrocytes integrate synaptic information. Furthermore, the modulation of the responses was observed also at the level of selected regions of the processes, supporting the idea that they represent the elementary units involved in neuron-astrocyte reciprocal communication (Grosche et al., 1999; Haydon and Araque, 2002; Nett et al., 2002; Fiacco and McCarthy, 2004). Finally, the simultaneous activity of different synapses also controlled the spatial extension of the intracellular $\mathrm{Ca}^{2+}$ signal.

The discrimination between glutamate released from SC terminals but not from glutamatergic axons in the alveus suggests the existence of astrocytic functional domains that grant a localized neuron-astrocyte communication resulting from a highly selective synaptic activation of astrocytic receptors. Although the underlying mechanism is unknown, it can be hypothesized that GluRs and transporters are specifically located in the membrane (e.g., GluRs may be placed close to SC terminals but not to glutamatergic axons in the alveus). This inhomogeneous spatial distribution of receptors and transporters may establish extracellular pathways that allow the selective activation of astrocytic GluRs by specific synaptic terminals. In the CA1 region of the hippocampus, this discrimination would promote feedforward versus feedback glutamatergic neuron-astrocyte communication, because although astrocytes responded to glutamatergic SCs (i.e., CA3 to CA1 signaling), they did not respond to glutamatergic axons in the alveus (e.g., CA1 to CA1 signaling provided by recurrent collaterals from CA1 pyramidal neurons). Therefore, astrocyte discrimination of neurotransmitters represents a cellular property that defines signaling pathways between neurons and astrocytes.

On the other hand, considering that the astrocyte $\mathrm{Ca}^{2+}$ can be selectively increased in different processes and that astrocytes release glutamate through a $\mathrm{Ca}^{2+}$-dependent mechanism, the discrimination between two different transmitters (glutamate and $\mathrm{ACh}$ ) grants a specific spatial distribution of the $\mathrm{Ca}^{2+}$ dependent neuromodulatory effects on different synapses. For instance, although during theta rhythm both glutamatergic and cholinergic terminals from axons originated in the septum and diagonal band of Broca might be active, $\mathrm{Ca}^{2+}$ would increase only in astrocytic processes that respond to ACh, and only synapses close to those processes would be selectively modulated (unless the $\mathrm{Ca}^{2+}$ signal propagates along the astrocyte), whereas synapses close to glutamate-responding processes would be unaffected.

The ability of most neurotransmitters to increase astrocytic
$\mathrm{Ca}^{2+}$ levels is firmly established (Porter and McCarthy, 1997; Verkhratsky et al., 1998). Recent reports have shown that astrocytic receptor activation by exogenously applied transmitters may have synergistic effects that increase the $\mathrm{Ca}^{2+}$ signal (Fatatis et al., 1994; Cormier et al., 2001; Sul et al., 2004). Although previous studies only reported synaptic-induced increases or synergistic enhancements of the astrocytic $\mathrm{Ca}^{2+}$ signal, the existence of neuronal signaling that reduces $\mathrm{Ca}^{2+}$ levels was unknown. Here we show that the simultaneous activity of cholinergic and glutamatergic synapses may induce the relative reduction of the $\mathrm{Ca}^{2+}$ signal, which indicates that negative cooperative actions of neurotransmitters may occur and that the astrocytic $\mathrm{Ca}^{2+}$ signal is susceptible of relative depression, which might represent a form of inhibition. Therefore, the existence of positive and negative cooperative actions of neurotransmitters confers a higher degree of complexity to the properties of the information transfer between neurons and astrocytes.

Numerous $\mathrm{Ca}^{2+}$-dependent cellular phenomena may be regulated by the astrocyte $\mathrm{Ca}^{2+}$ signal modulation. Regarding astrocyte-neuron communication, it may be crucial in the regulation of $\mathrm{Ca}^{2+}$-dependent release of transmitters, such as glutamate, and therefore in their neuromodulatory effects. Furthermore, although low-frequency synaptic activity usually leads to $\mathrm{Ca}^{2+} \mathrm{el}-$ evations that remain restricted to individual astrocytic processes, high-frequency evoked $\mathrm{Ca}^{2+}$ elevations initiate in specific processes and then propagate to the soma and other processes (Porter and McCarthy, 1996; Pasti et al., 1997; Kang et al., 1998; Grosche et al., 1999). This has been proposed to be the basis of a code for neuron-astrocyte communication that is decoded into feedback and feedforward signals that define the spatial extension of astrocyte-induced neuromodulation (Fellin and Carmignoto, 2004). Present findings, however, show that the astrocyte $\mathrm{Ca}^{2+}$ modulation controls the intracellular propagation of the $\mathrm{Ca}^{2+}$ signal, indicating that the characteristics of the neuron-astrocyte communication is not only determined by the synaptic activity level but also by the spatiotemporal features of the activity of the different incoming synaptic inputs. Furthermore, considering the demonstration that astrocyte $\mathrm{Ca}^{2+}$ evokes NMDARmediated currents in CA1 pyramidal neurons, which supports the neuromodulatory role of glutamate released by astrocytes, the synaptic control of the $\mathrm{Ca}^{2+}$ signal propagation may have important consequences on brain function by regulating the spatial range of astrocyte influence on synaptic terminals and cerebral microcirculation.

Although we did not attempt to determine the cellular mechanisms responsible for the $\mathrm{Ca}^{2+}$ signal modulation, current data suggest that this mechanism does not rely on neural network interaction, rather it results from regulatory phenomena at the intracellular level, supporting the existence of cellular intrinsic properties. Although we cannot discard the existence of undetected $\mathrm{Ca}^{2+}$ signal, the fact that this regulation did not seem to require detectable $\mathrm{Ca}^{2+}$ elevations evoked by both pathways suggests that the limiting factor modulating the $\mathrm{Ca}^{2+}$ signal may not depend on the $\mathrm{Ca}^{2+}$ itself and that it resides upstream the $\mathrm{Ca}^{2+}$ release from the internal stores. Nevertheless, additional studies aimed at determining the possible interaction between the intracellular signaling pathways are required to elucidate the underlying molecular mechanism.

$\mathrm{Ca}^{2+}$ elevations in astrocytes stimulate the release of glutamate, which acting on presynaptic or postsynaptic receptors modulates synaptic transmission and neuronal excitability (Araque et al., 1998a,b; Kang et al., 1998; Parri et al., 2001; Pasti et al., 2001; Brockhaus and Deitmer, 2002; Fiacco and McCarthy, 
2004; Liu et al., 2004). Here we show that $\mathrm{Ca}^{2+}$ elevations in astrocytes induced slowly developing and long-lasting NMDARdependent currents in hippocampal pyramidal neurons. Similar currents, originally described in cultured cells and termed SIC (Araque et al., 1998a), have been recorded during spontaneous astrocytic $\mathrm{Ca}^{2+}$ elevations in thalamic neurons, where they are thought to play some role in neural network maturation (Parri et al., 2001). Furthermore, while the present manuscript was in preparation, two recent articles have reported the presence of neuronal NMDAR-mediated SICs that can be triggered by stimuli that evoke $\mathrm{Ca}^{2+}$ elevations in astrocytes (Angulo et al., 2004; Fellin et al., 2004) and that can serve to synchronize neuronal activity. Our results describing the cellular mechanisms of the astrocyte-induced SICs in neurons are in close agreement with those findings. The differences between the mean SIC amplitude described here and that reported by Fellin et al. (2004) may partially be attributable to the different experimental conditions. Indeed, although this group analyzed SICs after strong SC stimulation or perfusion with $(R S)$-3,5-dihydroxyphenylglycine (i.e., when many astrocytes were activated), we studied SICs that occurred spontaneously or after depolarization of a single astrocyte, suggesting that the SICs reported here correspond to unitary events. Consistent with this idea, we show that local application of ATP, which stimulates numerous astrocytes (Fig. 7), evoked SICs of higher amplitude. Therefore, the different number of stimulated astrocytes in the different conditions could account for the observed differences in the SIC amplitudes. Nevertheless, the mean amplitude of the spontaneous SICs reported by Fellin et al. (2004) is also significantly higher than the mean amplitude of those described here. The origin of this difference is unknown but might be simply attributable to different detection criteria. Nevertheless, the similar pharmacological and kinetic properties of the spontaneous SICs recorded by Fellin et al. (2004) and by us suggest that they correspond to the same type of events.

The occurrence of SICs during a synaptic-evoked astrocyte $\mathrm{Ca}^{2+}$ signal was not investigated because nerve stimulation could directly induce neuronal responses that could be erroneously identified as astrocyte-mediated SICs. Therefore, these SICs are not necessarily linked to a neuron-evoked $\mathrm{Ca}^{2+}$ signal. Although it might seem likely that stimuli that evoked $\mathrm{Ca}^{2+}$ elevations may lead to similar consequences, additional studies are required to characterize the dependence of SICs on a neuron-evoked astrocyte $\mathrm{Ca}^{2+}$ signal.

Although we did not explore the physiological meaning of the SICs reported here, the fact that they represent NMDARmediated responses, suggests that, besides the reported neuronal synchronization, the astrocyte-neuron communication may serve to the postsynaptic neuron as a coincidence detection mechanism between synaptic and astrocytic signaling.

Cholinergic transmission plays an important role in the physiology of the hippocampus, being involved in the generation of the hippocampal theta rhythm and in some forms of synaptic plasticity, such as long-term potentiation, by interacting with glutamatergic transmission (Auerbach and Segal, 1996; Buzsaki, 2002). The current demonstration that the astrocyte $\mathrm{Ca}^{2+}$ signal is modulated by the simultaneous activity of both glutamatergic and cholinergic synapses suggests that astrocytes might participate in these phenomena. Furthermore, we show that astrocytic $\mathrm{Ca}^{2+}$ signal is potentiated when both types of synaptic terminals are coincidentally active at relative low frequencies (between 1 and 10 $\mathrm{Hz}$ ) that are in the range of the theta rhythm. Therefore, it is tempting to speculate that astrocytes may act as coincidence detector elements potentiating their intracellular $\mathrm{Ca}^{2+}$ signal during the theta rhythm, being thus possibly involved in this functional state of the hippocampus.

In conclusion, the ability of astrocytes to discriminate between the activity of different synapses belonging to different axon pathways, the bidirectional modulation of the astrocytic cellular excitability by the synaptic activity, and the expression of cellular intrinsic properties indicate that astrocytes are endowed with cellular computational characteristics that integrate synaptic information. Therefore, in addition to neurons, astrocytes could also be considered as cellular elements involved in the information processing by the nervous system.

\section{References}

Aguado F, Espinosa-Parrilla JF, Carmona MA, Soriano E (2002) Neuronal activity regulates correlated network properties of spontaneous calcium transients in astrocytes in situ. J Neurosci 22:9430-9444.

Angulo MC, Kozlov AS, Charpak S, Audinat E (2004) Glutamate released from glial cells synchronizes neuronal activity in the hippocampus. J Neurosci 24:6920-6927.

Araque A, Parpura V, Sanzgiri RP, Haydon PG (1998a) Glutamatedependent astrocyte modulation of synaptic transmission between cultured hippocampal neurons. Eur J Neurosci 10:2129-2142.

Araque A, Sanzgiri RP, Parpura V, Haydon PG (1998b) Calcium elevation in astrocytes causes an NMDA receptor-dependent increase in the frequency of miniature synaptic currents in cultured hippocampal neurons. J Neurosci 18:6822-6829.

Araque A, Parpura V, Sanzgiri RP, Haydon PG (1999) Tripartite synapses: glia, the unacknowledged partner. Trends Neurosci 22:208-215.

Araque A, Li N, Doyle RT, Haydon PG (2000) SNARE protein-dependent glutamate release from astrocytes. J Neurosci 20:666-673.

Araque A, Martin ED, Perea G, Arellano JI, Buño W (2002) Synaptically released acetylcholine evokes $\mathrm{Ca}^{2+}$ elevations in astrocytes in hippocampal slices. J Neurosci 22:2443-2450.

Auerbach JM, Segal M (1996) Muscarinic receptors mediating depression and long-term potentiation in rat hippocampus. J Physiol (Lond) 492:479-493.

Auld DS, Robitaille R (2003) Glial cells and neurotransmission: an inclusive view of synaptic function. Neuron 40:389-400.

Beattie EC, Stellwagen D, Morishita W, Bresnahan JC, Ha BK, Von Zastrow M, Beattie MS, Malenka RC (2002) Control of synaptic strength by glial TNF $\alpha$. Science 295:2282-2285.

Bergles DE, Jahr CE (1997) Synaptic activation of glutamate transporters in hippocampal astrocytes. Neuron 19:1297-1308.

Bergles DE, Diamond JS, Jahr CE (1999) Clearance of glutamate inside the synapse and beyond. Curr Opin Neurobiol 9:293-298.

Bergles DE, Roberts JD, Somogyi P, Jahr CE (2000) Glutamatergic synapses on oligodendrocyte precursor cells in the hippocampus. Nature 405:187-191.

Bezzi P, Carmignoto G, Pasti L, Vesce S, Rossi D, Rizzini B, Pozzan T, Volterra A (1998) Prostaglandins stimulate $\mathrm{Ca}^{2+}$-dependent glutamate release in astrocytes. Nature 391:281-285.

Bezzi P, Gundersen V, Galbete JL, Seifert G, Steinhauser C, Pilati E, Volterra A (2004) Astrocytes contain a vesicular compartment that is competent for regulated exocytosis of glutamate. Nat Neurosci 7:613-620.

Brockhaus J, Deitmer JW (2002) Long-lasting modulation of synaptic input to Purkinje neurons by Bergmann glia stimulation in rat brain slices. J Physiol (Lond) 545:581-593.

Buzsaki G (2002) Theta oscillations in the hippocampus. Neuron 33:325-340.

Carmignoto G (2000) Reciprocal communication systems between astrocytes and neurons. Prog Neurobiol 62:561-581.

Charles AC, Giaume C (2002) Intercellular calcium waves in astrocytes: underlying mechanisms and functional significance. In: The tripartite synapse: glia in synaptic transmission (Volterra A, Magistretti PJ, Haydon PG, eds), pp 110-126. New York: Oxford UP.

Cormier RJ, Mennerick S, Melbostad H, Zorumski CF (2001) Basal levels of adenosine modulate mGluR5 on rat hippocampal astrocytes. Glia 33:24-35.

Fatatis A, Holtzclaw LA, Avidor R, Brenneman DE, Russell JT (1994) Vasoactive intestinal peptide increases intracellular calcium in astroglia: syn- 
ergism with alpha-adrenergic receptors. Proc Natl Acad Sci USA 91:2036-2040.

Fellin T, Carmignoto G (2004) Neuron-to-astrocyte signaling in the brain represents a distinct multifunctional unit. J Physiol (Lond) 559:3-15.

Fellin T, Pascual O, Gobbo S, Pozzan T, Haydon PG, Carmignoto G (2004) Neuronal synchrony mediated by astrocytic glutamate through activation of extrasynaptic NMDA receptors. Neuron 43:729-743.

Fernández de Sevilla D, Buño W (2003) Presynaptic inhibition of Schaffer collateral synapses by stimulation of hippocampal cholinergic afferent fibers. Eur J Neurosci 17:555-558.

Fiacco TA, McCarthy KD (2004) Intracellular astrocyte calcium waves in situ increase the frequency of spontaneous AMPA receptor currents in CA1 pyramidal neurons. J Neurosci 24:722-732.

Grosche J, Matyash V, Moller T, Verkhratsky A, Reichenbach A, Kettenmann H (1999) Microdomains for neuron-glia interaction: parallel fiber signaling to Bergmann glial cells. Nat Neurosci 2:139-143.

Haydon PG (2001) Glia: listening and talking to the synapse. Nat Rev Neurosci 2:185-193.

Haydon PG, Araque A (2002) Astrocytes as modulators of synaptic transmission. In: The tripartite synapse: glia in synaptic transmission (Volterra A, Magistretti PJ, Haydon PG, eds), pp 185-198. New York: Oxford UP.

Kang J, Jiang L, Goldman SA, Nedergaard M (1998) Astrocyte-mediated potentiation of inhibitory synaptic transmission. Nat Neurosci 1:683-692.

Latour I, Gee CE, Robitaille R, Lacaille JC (2001) Differential mechanisms of $\mathrm{Ca}^{2+}$ responses in glial cells evoked by exogenous and endogenous glutamate in rat hippocampus. Hippocampus 11:132-145.

Lewis PR, Shute CCD (1967) The cholinergic limbic system: projections to hippocampal formation, medial cortex, nuclei of the ascending cholinergic reticular system, and the subfornical organ and supra-optic crest. Brain 90:521-540.

Li X-G, Somogyi P, Ylinen A, Buzsaki G (1994) The hippocampal CA3 network: an in vivo intracellular labeling study. J Comp Neurol 339:181-208.

Liu QS, Xu Q, Arcuino G, Kang J, Nedergaard M (2004) Astrocyte-mediated activation of neuronal kainate receptors. Proc Natl Acad Sci USA 101:3172-3177.

McCormick DA (1998) Membrane properties and neurotransmitter actions. In: The synaptic organization of the brain (Shepherd GM, ed), pp 37-75. New York: Oxford UP.

Mennerick S, Benz A, Zorumski CF (1996) Components of glial responses to exogenous and synaptic glutamate in rat hippocampal microcultures. J Neurosci 16:55-64.

Nedergaard M, Ransom B, Goldman SA (2003) New roles for astrocytes: Redefining the functional architecture of the brain. Trends Neurosci 26:523-530.
Nett WJ, Oloff SH, McCarthy KD (2002) Hippocampal astrocytes in situ exhibit calcium oscillations that occur independent of neuronal activity. J Neurophysiol 87:528-537.

Newman EA (2003) New roles for astrocytes: regulation of synaptic function. Trends Neurosci 26:536-542.

Newman EA, Zahs KR (1998) Modulation of neuronal activity by glial cells in the retina. J Neurosci 18:4022-4028.

Parpura V, Haydon PG (2000) Physiological astrocytic calcium levels stimulate glutamate release to modulate adjacent neurons. Proc Natl Acad Sci USA 97:8629-8634.

Parri HR, Gould TM, Crunelli V (2001) Spontaneous astrocytic $\mathrm{Ca}^{2+}$ oscillations in situ drive NMDAR-mediated neuronal excitation. Nat Neurosci 4:803-812.

Pasti L, Volterra A, Pozzan T, Carmignoto G (1997) Intracellular calcium oscillations in astrocytes: a highly plastic, bidirectional form of communication between neurons and astrocytes in situ. J Neurosci 17:7817-7830.

Pasti L, Zonta M, Pozzan T, Vicini S, Carmignoto G (2001) Cytosolic calcium oscillations in astrocytes may regulate exocytotic release of glutamate. J Neurosci 21:477-484.

Porter JT, McCarthy KD (1996) Hippocampal astrocytes in situ respond to glutamate released from synaptic terminals. J Neurosci 16:5073-5081.

Porter JT, McCarthy KD (1997) Astrocytic neurotransmitter receptors in situ and in vivo. Prog Neurobiol 51:439-455.

Robitaille R (1998) Modulation of synaptic efficacy and synaptic depression by glial cells at the frog neuromuscular junction. Neuron 21:847-855.

Shelton MK, McCarthy KD (1999) Mature hippocampal astrocytes exhibit functional metabotropic and ionotropic glutamate receptors in situ. Glia 26:1-11.

Sul JY, Orosz G, Givens RS, Haydon PG (2004) Astrocytic connectivity in the hippocampus. Neuron Glia Biol 1:3-11.

Verkhratsky A, Orkand RK, Kettenmann H (1998) Glial calcium: homeostasis and signaling function. Physiol Rev 78:99-141.

Volterra A, Bezzi P (2002) Release of transmitters from glial cells. In: The tripartite synapse: glia in synaptic transmission (Volterra A, Magistretti PJ, Haydon PG, eds), pp 164-184. New York: Oxford UP.

Zhang J, Wang H, Ye C, Ge W, Chen Y, Jiang Z, Wu C, Poo M, Duan S (2003) ATP released by astrocytes mediates glutamatergic activity-dependent heterosynaptic suppression. Neuron 40:971-982.

Zhang Q, Fukuda M, Van Bockstaele E, Pascual O, Haydon PG (2004) Synaptotagmin IV regulates glial glutamate release. Proc Natl Acad Sci USA 101:9441-9446.

Zonta M, Angulo MC, Gobbo S, Rosengarten B, Hossmann KA, Pozzan T, Carmignoto G (2003) Neuron-to-astrocyte signaling is central to the dynamic control of brain microcirculation. Nat Neurosci 6:43-50. 